MPI-PhT 98-42

May 1998

\title{
On Nonlinear $\sigma$-Models arising in (Super-)Gravity
}

\author{
Peter Breitenlohner and Dieter Maison \\ Max-Planck-Institut für Physik \\ (Werner-Heisenberg-Institut) \\ Föhringer Ring 6, D-80805 München, Germany
}

\begin{abstract}
In a previous paper with Gibbons [1] we derived a list of three dimensional symmetric space $\sigma$-model obtained by dimensional reduction of a class of four dimensional gravity theories with abelian gauge fields and scalars. Here we give a detailed analysis of their group theoretical structure leading to an abstract parametrization in terms of 'triangular' group elements. This allows for a uniform treatment of all these models. As an interesting application we give a simple derivation of a 'Quadratic Mass Formula' for strictly stationary black holes.
\end{abstract}

\section{Introduction}

Starting from the maximal eleven dimensional supergravity theory many different models with $N \geq 1$ supersymmetries have been constructed in lower dimensions via Kałuza-Klein reductions. In the case of compactification on a torus the use of adapted coordinates leads to abelian gauge symmetries derived from the general covariance in eleven dimensions. Depending on the dimension some of the abelian vector resp. tensor gauge fields can be replaced by scalar potentials via Hodge duality. Experience shows that all the scalars of these models organize themselves into the simple structure of a non-linear sigma model with a coset space $G / H$ as target space. Even more, these coset spaces have the structure of a Riemannian resp. pseudo-Riemannian non-compact symmetric space. As the number of 
scalars increases when the dimension is reduced it reaches a maximum in three dimensions. Although further reduction to two dimensions results in another scalar from the 3-metric, the latter plays a different role and does not lead to a further increase of $G / H$. In our previous paper with Gibbons 11 we gave a general classification of four dimensional bosonic theories leading to three dimensional reductions allowing for Ehlers-Harrison type transformations. Besides the models obtained from supergravities we found many new cases including certain infinite series, all of them resulting in symmetric space sigma models. The present paper is devoted to an elaboration of the specific structure of all these coset spaces.

An essential step for the identification of the particular sigma model corresponding to a theory is the parametrization of the coset space with the scalar fields of the theory. Considering specific examples one finds a typical structure of the target space metric. While some of the fields appear only in polynomial form others do not. This has been exploited in the past to construct certain triangular matrix representations of the coset spaces, such that the polynomial dependence is related to the nilpotent (off-diagonal) part of the matrices. This reminds very much of the Iwasawa decomposition for semi-simple Lie groups resp. its reduction to the coset space. There is a corresponding structure in the action of the various transformations of $G$ on the fields, in particular the generalized 'Ehlers' and 'Harrison' transformations. In fact, this will be the starting point of our structural analysis of the Lie algebra $g$ of $G$. It turns out that all the different cases can be characterized by certain matrices made up from the strucure constants of $g$. As a result, we not only get the complete action of $g$ on the fields, but also obtain an abstract parametrization of the coset space $G / H$ in terms of the fields via the exponential map. Clearly, in order to obtain a concrete parametrization, the above mentioned matrices have to be worked out for each case resp. family of models individually and we shall exemplify this in detail. Nevertheless for certain applications our knowledge on the general structure of $G$ is sufficient without the need of any specific parametrization. A particularly interesting case is provided by the 'Quadratic Mass Formula' for strictly stationary single black hole solutions, generalizing work of Heusler [4]. Another important application we have in mind but not yet completely worked out is a proof of the uniqueness of the 'Generalized Kerr Solutions', which we announced already in [1].

In Chapter 2 we review the dimensional reduction from four to three

\footnotetext{
${ }^{1}$ There is however a dramatic increase of $G$ to the infinite dimensional Geroch group, if the complete integrability of the two dimensional theories is taken into account [2, 3].
} 
dimensions for stationary or axisymmetric solutions, concentrating on those cases, where the scalars of the 3-dimensional theory form a nonlinear $\sigma$ model, i.e. parametrize a pseudo Riemannian or Riemannian symmetric space and consequently there exist generalized Ehlers-Harrison transformations. We closely follow the treatment in [1] and for the convenience of the reader we repeat the list of all such cases from that paper. In Chapter 3 we analyze the general structure of the Lie algebra $g$ and derive consistency conditions for the coefficients used to parametrize the structure constants of the Lie algebra $g$ for all the possible cases. In Chapter 4 we discuss the parametrization of coset representatives forming a 'triangular' subgroup $T \subset G$, the action of (infinitesimal) Ehlers-Harrison transformations, and the form of the Lagrangian built from the Lie algebra valued conserved current $J$. Chapter 5 contains two simple applications for strictly stationary black holes. In the appendix we give a detailed discussion of all the cases.

\section{Dimensional Reduction from 4 to 3 Dimensions}

We consider 4-dimensional theories with scalars forming a nonlinear $\sigma$-model coupled to gravity and $k$ abelian gauge fields. Stationary solutions of the field equations can be described in terms of a 'dimensionally reduced' 3dimensional theory. We will closely follow the analysis in [1] and use a rather similar notation.

\subsection{The 4-Dimensional Theory}

We start from a Lagrangian field theory over a space-time manifold $\Sigma_{4}$ with coordinates $x^{a}$ and metric $g_{a b}(x)$ (with $d s^{2}=g_{a b} d x^{a} d x^{b}>0$ along time-like directions). Let $\bar{\Phi}$ be a Riemannian symmetric space with (real) coordinates $\bar{\phi}^{i}$ and metric $\bar{\gamma}_{i j}(\bar{\phi})$. The nonlinear $\sigma$-model with target space $\bar{\Phi}$ is characterized by the kinetic term $\frac{1}{2} g^{a b}(x) \partial_{a} \bar{\phi}^{i}(x) \partial_{b} \bar{\phi}^{j}(x) \bar{\gamma}_{i j}(\bar{\phi}(x))$. We are interested in particular in the case $\bar{\Phi}=\bar{G} / \bar{H}$, where $\bar{G}$ is a noncompact Lie group with maximal compact subgroup $\bar{H}$. We choose a basis $s_{i}, i=$ $1, \ldots, \operatorname{dim} \bar{G}$ for the Lie algebra $\bar{g}$ of $\bar{G}$ with commutators

$$
\left[s_{i}, s_{j}\right]=f_{i j}^{l} s_{l} .
$$

Due to the assumption that $\bar{G} / \bar{H}$ is a symmetric space, there exists an involutive automorphism $\bar{\tau}$ of $\bar{g}$

$$
\bar{\tau}([X, Y])=[\bar{\tau}(X), \bar{\tau}(Y)] \quad \text { for all } X, Y \in \bar{g}, \quad \bar{\tau}^{2}=\mathrm{Id},
$$


such that

$$
\bar{h}=\{X \in \bar{g}: \bar{\tau}(X)=X\} .
$$

We can describe elements of the coset space $\bar{G} / \bar{H}$ by coset representatives $\bar{\pi}(x) \in \bar{G}$. The group $\bar{G}$ acts on these representatives, but in addition there is the gauge group $\bar{H}$ changing the coset representatives

$$
\bar{\pi}(x) \mapsto \bar{v}(x) \bar{\pi}(x) \bar{u}^{-1}, \quad \text { with } \quad \bar{u} \in \bar{G}, \quad \bar{v}(x) \in H .
$$

In order to eliminate the gauge degrees of freedom we consider the gauge invariant group element $\bar{\mu}=\bar{\tau}\left(\bar{\pi}^{-1}\right) \bar{\pi}$ and the Lie algebra valued currents $\bar{J}=\bar{J}_{a} d x^{a}=\frac{1}{2} \bar{\mu}^{-1} d \bar{\mu}$. This allows us to rewrite the kinetic term for the scalars in the form $\left.\frac{1}{4}<\bar{J}_{a}, \bar{J}^{a}\right\rangle_{\bar{g}}$, where $<\cdot, \cdot>_{\bar{g}}$ is a suitable (not necessarily unique) invariant scalar product on the Lie algebra $\bar{g}$. Finally, let there be $k$ (real) abelian vector fields ('electric' potentials) $B_{a}=\left(B_{a}^{I}\right), I=1, \ldots, k$ with field strengths $G_{a b}=\partial_{a} B_{b}-\partial_{b} B_{a}$ and their duals ${ }^{*} G_{a b}$.

For the coupled system of gravity, scalars, and vector fields we choose the action

$$
S_{4}=\int_{\Sigma_{4}} \sqrt{|g|} d^{4} x\left(-\frac{1}{2} R+\frac{1}{4}<\bar{J}_{a}, \bar{J}^{a}>_{\bar{g}}-\frac{c}{8} G_{a b}^{T}\left(\tilde{\mu} G^{a b}-\tilde{\nu}^{*} G^{a b}\right)\right),
$$

where $R$ is the scalar curvature, $c$ is a positive constant, $\tilde{\mu}(\bar{\phi})$ and $\tilde{\nu}(\bar{\phi})$ are symmetric $k \times k$ matrices, and $\tilde{\mu}$ is positive definite. The constant $c$ could be absorbed by rescaling the matrices $\tilde{\mu}$ and $\tilde{\nu}$ and/or the vector fields $B_{a}$. Rescaling the matrices would, however, invalidate the identification of $\tilde{\mu}$ and $\bar{M}$ (defined below) as matrix representatives of elements of the group $\bar{G}$ (compare Chapter 4 ). Rescaling the vector fields may be equally undesirable; for the Kałuza-Klein theories discussed in detail in Section A.1 the vector fields $B_{a}^{I}$ are directly related to components of the metric in $4+n$ dimensions. For the generalized Einstein-Maxwell theories discussed in Section A.2 one would like to normalize the vector fields, and thus the charges, such that a Reissner-Nordstrøm black hole with charge $q$ has mass $m \geq|q|$.

The field equations for the vector fields $\nabla_{a}\left(\tilde{\mu} G^{a b}-\tilde{\nu}^{*} G^{a b}\right)=0$ can be interpreted as Bianchi identitities for field strengths $H_{a b}=\partial_{a} C_{b}-\partial_{b} C_{a}$ derived from 'magnetic' potentials $C_{a}$. Choosing ${ }^{*} H_{a b}=\eta\left(\tilde{\mu} G_{a b}-\tilde{\nu}^{*} G_{a b}\right)$ with some constant orthogonal matrix $\eta$, we obtain $2 k$ vector fields $A_{a}$ and field strengths $F_{a b}=\partial_{a} A_{b}-\partial_{b} A_{a}$ satisfying the linear relation

$$
F_{a b}=\bar{Y} \bar{M}^{*} F_{a b}, \quad F_{a b}=\left(\begin{array}{c}
G_{a b} \\
H_{a b}
\end{array}\right), \quad A_{m}=\left(\begin{array}{c}
B_{m} \\
C_{m}
\end{array}\right),
$$


with matrices $\bar{M}=\bar{M}^{T}$ and $\bar{Y}=-\bar{Y}^{T}$ such that $\bar{Y} \bar{M} \bar{Y}=-\bar{M}^{-1}$

$$
\bar{Y}=\left(\begin{array}{cc}
0 & \eta^{T} \\
-\eta & 0
\end{array}\right), \quad \bar{M}=\left(\begin{array}{cc}
\tilde{\mu}+\tilde{\nu} \tilde{\mu}^{-1} \tilde{\nu} & \tilde{\nu} \tilde{\mu}^{-1} \eta^{T} \\
\eta \tilde{\mu}^{-1} \tilde{\nu} & \eta \tilde{\mu}^{-1} \eta^{T}
\end{array}\right)
$$

Assume there is a $2 k$-dimensional real matrix representation $\bar{\rho}$ of $\bar{G}$ with

$$
\bar{\rho}: \bar{\pi} \mapsto \bar{\rho}(\bar{\pi})=\bar{P}, \quad \bar{\rho}(\bar{\tau}(\bar{\pi}))=\bar{P}^{T-1}, \quad \bar{\rho}(\bar{\mu})=\bar{P}^{T} \bar{P}=\bar{M} .
$$

The field equations for the vectors are then $\bar{G}$ invariant, provided the action of $\bar{G}$ on the field strenths is $\bar{G} \ni \bar{u}: F_{a b} \mapsto \bar{\rho}(\bar{u}) F_{a b}$. Thus the group $\bar{G}$

acts nonlinearly on the scalars $\bar{\phi}$, but acts linearly on the field strengths $G_{a b}$ and their duals ${ }^{*} G_{a b}$ (with coefficient depending on the scalars). The contribution of the vector fields to the gravitational and scalar field equations can be similarly expressed in terms of $F_{a b}$ in explicitly $\bar{G}$-covariant form. This 'on-shell' symmetry can, however, in general not be formulated as an invariance of the action.

\subsection{The 3-Dimensional Theory}

A solution of the 4-dimensional field equations with a 1-parameter symmetry group is characterized by a Killing vector field $K$ such that the Lie derivative with respect to $K$ of the metric, scalars, and field strengths vanishes. The solution is stationary, strictly stationary, or axisymmetric if $K$ is asymptotically time-like, everywhere time-like, or asymptotically spacelike respectively. In the following we will mostly assume that the solution is strictly stationary, i.e. $\Delta=K_{a} K^{a}>0$, but the results hold as well for axisymmetric solutions with $\Delta<0$.

Although the dimensional reduction from 4 to 3 dimensions could be formulated in a coordinate independent form, the discussion is simplified by choosing adapted coordinates such that the isometry is just a translation (e.g. $x^{a}=\left(x^{m}, t\right)$ with $\left.K=\frac{\partial}{\partial t}\right)$. The metric, scalars, and field strengths will then depend only on the three coordinates $x^{m}, m=1,2,3$ parametrizing the orbit space $\Sigma_{3}$ of the action of $K$; in a suitable gauge this will also be true for the vector fields. We decompose the metric and vector fields into pieces perpendicular and parallel to $K_{a}=\left(\Delta k_{m}, \Delta\right)$

$$
g_{a b}=\left(\begin{array}{cc}
-\frac{1}{\Delta} h_{m n}+\Delta k_{m} k_{n} & \Delta k_{n} \\
\Delta k_{m} & \Delta
\end{array}\right), \quad B_{a}=\left(\begin{array}{c}
\tilde{B}_{m}+B k_{m} \\
B
\end{array}\right) .
$$

Inserting this decomposition into the action $S_{4}$ and omitting the integration over $d t$ as well as surface terms, we obtain the action $S_{3}$ describing a 
Lagrangian field theory over the orbit space $\Sigma_{3}$, the dimensionally reduced theory. The action $\Sigma_{3}$ depends on the 3 -vectors $k_{m}$ and $\tilde{B}_{m}$ only through their field strengths $k_{m n}=\partial_{m} k_{n}-\partial_{n} k_{m}$ and $\tilde{B}_{m n}=\partial_{m} \tilde{B}_{n}-\partial_{n} \tilde{B}_{m}$; the resulting field equations can again be interpreted as consistency conditions for the existence of dual potentials. For ${ }^{*} \tilde{B}_{m n}$ these are just the parallel components $C$ of $C_{a}$, thus the $k$ vectors $B_{a}$ of the 4-dimensional theory yield $2 k$ scalars $A$. The 'twist' ${ }^{*} k_{m n}$ can be expressed in terms of the 'twist vector' $\omega_{m}$ with the 'twist potential' $\psi$

$$
A=\left(\begin{array}{c}
B \\
C
\end{array}\right), \quad \omega_{m}=\partial_{m} \psi+\frac{c}{2} A^{T} \bar{Y}^{-1} \partial_{m} A .
$$

Performing these dualizations via Lagrange multipliers finally yields the dimensionally reduced action in the form

$$
\begin{gathered}
S_{3}=\int_{\Sigma_{3}} \sqrt{|h|} d^{3} x\left(\frac{1}{2} R-\frac{1}{4}<\bar{J}_{m}, \bar{J}^{m}>_{\bar{g}}+\frac{c}{4 \Delta} \partial_{m} A^{T} \bar{M} \partial^{m} A\right. \\
\left.-\frac{1}{4 \Delta^{2}}\left(\partial_{m} \Delta \partial^{m} \Delta+\omega_{m} \omega^{m}\right)\right)
\end{gathered}
$$

where the 3-metric $h_{m n}$ and its inverse are used to compute the scalar curvature $R$ as well as to raise indices. We have used the rescaled 3-metric $h_{m n}$, assuming $\Delta \neq 0$, in order to obtain an action without additional coefficient for the scalar curvature.

The action $S_{3}$ describes a nonlinear $\sigma$-model with target space $\Phi$ coupled to 3-dimensional gravity (note, however, that 3-dimensional gravity has no dynamical degrees of freedom). $\Phi$ is a homogeneous space parametrized by $(\operatorname{dim} \bar{G}-\operatorname{dim} \bar{H})+2 k+2$ scalars $\phi=(\bar{\phi}, A, \Delta, \psi)$. The theory is invariant under various transformation (with constant parameters):

(1) twist gauge transformations $\psi \rightarrow \psi+\chi$,

(2) 'electromagnetic' gauge transformations $A \rightarrow A+\alpha, \psi \rightarrow \psi+\frac{c}{2} A^{T} \bar{Y}^{-1} \alpha$,

(3) scale transformations $A \rightarrow \sqrt{\zeta} A, \Delta \rightarrow \zeta \Delta, \psi \rightarrow \zeta \psi$, and

(4) $\bar{G}$ transformations acting on $\bar{\phi}$ and $A$.

The field equations for the scalar fields are equivalent to the conservation laws for corresponding Noether currents.

The 3-metric $h_{m n}$ is positive definite for $\Delta>0$ (strict stationarity) whereas $\Phi$ has an indefinite metric whith $2 k$ negative eigenvalues. For the axisymmetric case we will use primed variables $\Phi^{\prime}=\left(\bar{\phi}, A^{\prime}, \Delta^{\prime}, \psi^{\prime}\right)$ with $\Delta^{\prime}<0$; the metric on $\Phi^{\prime}$ is positive definite but the 3 -metric $h_{m n}^{\prime}$ has signature $(+,-,-)$. The restriction of $\Phi$ (or $\Phi^{\prime}$ ) to the submanifold $A=0$ (or $A^{\prime}=0$ ) is the symmetric space $\bar{G} / \bar{H} \otimes S L(2) / S O(2)$, invariant under 
the well known (infinitesimal) Ehlers transformation $\delta \bar{\phi}=0, \delta \Delta=2 \Delta \psi$, $\delta \psi=\psi^{2}-\Delta^{2}[5]$.

For some such theories the Ehlers transformation can be extended to an invariance of the whole target space $\Phi$ or $\Phi^{\prime}$; commuting this generalized Ehlers transformation with (infinitesimal) electromagnetic gauge transformations finally yields generalized Harrison transformations [6]. All these transformations form a noncompact Lie group $G$ with maximal compact subgroup $H^{\prime}$ and the target space is either the Riemannian symmetric space $\Phi^{\prime}=G / H^{\prime}$ or the pseudo Riemannian symmetric space $\Phi=G / H$, where $H$ is a noncompact real form of $H^{\prime}$. The dimensions of $G$ and $H$ are

$$
\operatorname{dim} G=\operatorname{dim} \bar{G}+\operatorname{dim} S L(2)+4 k, \quad \operatorname{dim} H=\operatorname{dim} \bar{H}+\operatorname{dim} S O(2)+2 k .
$$

The dimensionally reduced action can then be expressed in the form

$$
S_{3}=\int_{\Sigma_{3}} \sqrt{|h|} d^{3} x\left(\frac{1}{2} R+L\right), \quad \text { with } \quad L=-\frac{1}{4}<J_{m}, J^{m}>,
$$

where $\langle\cdot, \cdot\rangle$ is a suitably normalized invariant scalar product on the Lie Algebra $g$ of $G$.

The $G / H \sigma$-model is characterized by two commuting involutive automorphisms $\tau$ and $\tau^{\prime}$ : All elements of $G$ that are invariant under $\tau$ form the subgroup $H$, all elements invariant under $\tau^{\prime}$ form the subgroup $H^{\prime}$, and all elements invariant under $\tau \tau^{\prime}$ form the subgroup $\bar{G} \otimes S L(2)$. The restriction of $\tau$ or $\tau^{\prime}$ to $\bar{G}$ is the original automorphism $\bar{\tau}$; the restriction to $S L(2)$ is the automorphism defining the maximal compact subgroup $S O(2)$. We choose a basis $t_{i}$ for the Lie algebra $\operatorname{sl}(2)$, where $t_{+}=e, t_{0}=d$, and $t_{-}=k$ satisfy the commutation relations

$$
[d, e]=e, \quad[d, k]=-k, \quad[e, k]=2 d,
$$

and

$$
\tau(e)=-k, \quad \tau(d)=-d, \quad \tau(k)=-e .
$$

We may assume that the representation $\bar{\rho}$ of $\bar{G}$ is faithful, i.e., that all scalars of the 4-dimensional $\bar{G} / \bar{H} \sigma$-model couple to the vector fields. The group $G$ will then be simple, and Table 1 (a reproduction of Table 2 in [1]) lists all possible cases.

\section{Parametrization of the Lie Algebra}


Table 1: List of all symmetric spaces obtained by dimensional reduction from four to three dimensions of theories with scalars and vectors (reproduced from Table 2 in [1]).

\begin{tabular}{|c|c|c|c|c|}
\hline \# & $G / H$ & $\bar{G} / \bar{H}$ & $\operatorname{dim} \bar{G} / \bar{H}$ & $k$ \\
\hline 1 & $S L(n+2) / S O(n, 2)$ & $G L(n) / S O(n)$ & $\frac{n(n+1)}{2}$ & $n$ \\
\hline 2 & $\frac{S U(p+1, q+1)}{S(U(p, 1) \times U(1, q)}$ & $U(p, q) /(U(p) \times U(q))$ & $2 p q$ & $p+q$ \\
\hline 3 & $\frac{S O(p+2, q+2)}{S O(p, 2) \times S O(2, q)}$ & $\frac{S O(p, q)}{S O(p) \times S O(q)} \times \frac{S O(2,1)}{S O(2)}$ & $p q+2$ & $p+q$ \\
\hline 4 & $S O^{*}(2 n+4) / U(n, 2)$ & $\frac{S O^{*}(2 n)}{U(n)} \times \frac{S U(2)}{S U(2)}$ & $n(n-1)$ & $2 n$ \\
\hline 5 & $S p(2 n+2 ; \mathbf{R}) / U(n, 1)$ & $S p(2 n ; \mathbf{R}) / U(n)$ & $n(n+1)$ & $n$ \\
\hline 6 & $\frac{G_{2(+2)}}{S U(1,1) \times S U(1,1)}$ & $S U(1,1) / U(1)$ & 2 & 2 \\
\hline 7 & $\frac{F_{4(+4)}}{S p(6 ; \mathbf{R}) \times S U(1,1)}$ & $S p(6 ; \mathbf{R}) / U(3)$ & 12 & 7 \\
\hline 8 & $E_{6(+6)} / S p(8 ; \mathbf{R})$ & $S L(6) / S O(6)$ & 20 & 10 \\
\hline 9 & $\frac{E_{6(-2)}}{S U(3,3) \times S U(1,1)}$ & $\frac{S U(3,3)}{S(U(3) \times U(3))}$ & 18 & 10 \\
\hline 10 & $\frac{E_{6(-14)}}{S O^{*}(10) \times S O(2)}$ & $S U(5,1) / U(5)$ & 10 & 10 \\
\hline 11 & $E_{7(+7)} / S U(4,4)$ & $\frac{S O(6,6)}{S O(6) \times S O(6)}$ & 36 & 16 \\
\hline 12 & $\frac{E_{7(-5)}}{S O^{*}(12) \times S O(2,1)}$ & $S O^{*}(12) / U(6)$ & 30 & 16 \\
\hline 13 & $\frac{E_{7(-25)}}{E_{6(-14)} \times S O(2)}$ & $\frac{S O(10,2)}{S O(10) \times S O(2)}$ & 20 & 16 \\
\hline 14 & $E_{7(+8)} / S O^{*}(16)$ & $E_{7(+7)} / S U(8)$ & 70 & 28 \\
\hline 15 & $\frac{E_{8(-24)}}{E_{7(-25)} \times S U(1,1)}$ & $\frac{E_{7(-25)}}{E_{6(-78)} \times S O(2)}$ & 54 & 28 \\
\hline
\end{tabular}




\subsection{Commutation Relations}

We choose a basis for the Lie algebra $g$ of $G$ consisting of the generators $s_{i}$ of $\bar{g}$, the generators $t_{i}$ of $s l(2)$, and $4 k$ additional generators $h_{i}, a_{i}, i=1, \ldots, 2 k$ transforming as doublet under $s l(2)$

$$
\begin{array}{lll}
{\left[d, h_{i}\right]=\frac{1}{2} h_{i},} & {\left[e, h_{i}\right]=0,} & {\left[k, h_{i}\right]=-a_{i},} \\
{\left[d, a_{i}\right]=-\frac{1}{2} a_{i},} & {\left[e, a_{i}\right]=-h_{i},} & {\left[k, a_{i}\right]=0,}
\end{array}
$$

and with a $2 k$-dimensional real matrix representation $\bar{\rho}$ of $\bar{g}$

$$
\bar{\rho}: s_{i} \mapsto \bar{\rho}\left(s_{i}\right)=R_{i}, \quad\left[s_{i}, h \cdot \alpha\right]=h \cdot R_{i} \alpha, \quad\left[s_{i}, a \cdot \alpha\right]=a \cdot R_{i} \alpha .
$$

The remaining commutators can be determined from the Jacobi identities $J(x, y, z) \equiv[[X, Y], Z]+[[Y, Z], X]+[[Z, X], Y]=0:$

$$
\begin{aligned}
& J(h \cdot \alpha, h \cdot \beta, d) \Rightarrow[h \cdot \alpha, h \cdot \beta]=\alpha \cdot y \beta e, \\
& J(h \cdot \alpha, h \cdot \beta, k) \Rightarrow[h \cdot \alpha, a \cdot \beta]=\alpha \cdot y \beta d+\alpha \cdot x^{l} \beta s_{l}, \\
& J(h \cdot \alpha, a \cdot \beta, k) \Rightarrow[a \cdot \alpha, a \cdot \beta]=-\alpha \cdot y \beta k,
\end{aligned}
$$

with $y^{T}=-y$ and $x^{l T}=x^{l}$. Finally

$$
\begin{aligned}
& J\left(h \cdot \alpha, a \cdot \beta, s_{i}\right) \Rightarrow y R_{i}+R_{i}^{T} y=0, \quad x^{l} R_{i}+R_{i}^{T} x^{l}=f_{i j}{ }^{l} x^{j} \\
& J(h \cdot \alpha, h \cdot \beta, a \cdot \gamma) \Rightarrow \frac{1}{2} \alpha(\beta \cdot y \gamma)+R_{l} \alpha\left(\beta \cdot x^{l} \gamma\right)-(\alpha \leftrightarrow \beta)=\gamma(\alpha \cdot y \beta) .
\end{aligned}
$$

The trace of this 'completeness relation' yields

$$
x^{l} R_{l}-\left(x^{l} R_{l}\right)^{T}+(2 k+1) y=0 .
$$

\subsection{The Invariant Scalar Product}

The simple Lie algebra $g$ has the invariant scalar product (unique up to an overall factor)

$$
<X, Y>_{g}=\frac{1}{c_{g}} \operatorname{Tr}_{g}(\operatorname{Ad}(X) \operatorname{Ad}(Y)), \quad X, Y \in g,
$$

similarly the invariant scalar product on $s l(2)$ is

$$
<X, Y>_{s l(2)}=\frac{1}{c_{s l(2)}} \operatorname{Tr}_{s l(2)}(\operatorname{Ad}(X) \operatorname{Ad}(Y)), \quad X, Y \in \operatorname{sl}(2),
$$

where the action of the adjoint representation is

$$
\operatorname{Ad}(X)|Y\rangle=|[X, Y]\rangle, \quad X, Y \in g .
$$


In order to compute the scalar product for $\operatorname{sl}(2)$ we need the commutators

$$
\begin{aligned}
& {[d,[d, e]]=e, \quad[d,[d, d]]=0, \quad[d,[d, k]]=k \quad \Rightarrow<d, d>_{s l(2)}=\frac{2}{c_{s l(2)}},} \\
& {[e,[k, e]]=2 e, \quad[e,[k, d]]=2 d, \quad[e,[k, k]]=0 \Rightarrow<e, k>_{s l(2)}=\frac{4}{c_{s l(2)}} .}
\end{aligned}
$$

Choosing $c_{s l(2)}=2$ we obtain for $X=\epsilon e+\delta d+\kappa k$

$$
<X, X>_{s l(2)}=\delta^{2}+4 \epsilon \kappa=\delta^{2}+(\epsilon+\kappa)^{2}-(\epsilon-\kappa)^{2} .
$$

Extending the trace over all generators of $g$

$$
\begin{aligned}
& {[d,[d, h \cdot \alpha]]=\frac{1}{4} h \cdot \alpha, \quad[d,[d, a \cdot \alpha]]=\frac{1}{4} a \cdot \alpha \Rightarrow<d, d>_{g}=\frac{k+2}{c_{g}},} \\
& {[e,[k, h \cdot \alpha]]=h \cdot \alpha, \quad[e,[k, a \cdot \alpha]]=0 \quad \Rightarrow<e, k>_{g}=\frac{2(k+2)}{c_{g}} .}
\end{aligned}
$$

We choose $c_{g}=k+2$ such that the scalar product $\langle\cdot, \cdot\rangle_{s l(2)}$ coincides with the restriction of $\langle\cdot, \cdot\rangle_{g}$ to the subalgebra $s l(2)$. The restriction of $<\cdot, \cdot\rangle_{g}$ to the subalgebra $\bar{g}$ similarly defines a particular invariant scalar product on $\bar{g}$ (among possibly different ones when $\bar{g}$ is not simple). For the scalar product $\left\langle s_{i}, s_{j}\right\rangle$ we need the commutators

$$
\left[s_{i},\left[s_{j}, h \cdot \alpha\right]\right]=h \cdot R_{i} R_{j} \alpha, \quad\left[s_{i},\left[s_{j}, a \cdot \alpha\right]\right]=a \cdot R_{i} R_{j} \alpha,
$$

and find

$$
<s_{i}, s_{j}>=\frac{\operatorname{Tr}_{\bar{g}}\left(\operatorname{Ad}\left(s_{i}\right) \operatorname{Ad}\left(s_{j}\right)\right)+2 \operatorname{Tr}\left(R_{i} R_{j}\right)}{k+2} .
$$

For the scalar product $\langle h \cdot \alpha, a \cdot \beta\rangle$ we need the commutators

$$
\begin{aligned}
& {[h \cdot \alpha,[a \cdot \beta, e]]=(\alpha \cdot y \beta) e} \\
& {[h \cdot \alpha,[a \cdot \beta, h \cdot \gamma]]=h \cdot R_{l} \alpha\left(\beta \cdot x^{l} \gamma\right)-\frac{1}{2} h \cdot \alpha(\beta \cdot y \gamma)} \\
& {[h \cdot \alpha,[a \cdot \beta, d]]=\frac{1}{2}(\alpha \cdot y \beta) d+\frac{1}{2}\left(\alpha \cdot x^{l} \beta\right) s_{l}} \\
& {\left[h \cdot \alpha,\left[a \cdot \beta, s_{i}\right]\right]=-\left(\alpha \cdot y R_{i} \beta\right) d-\left(\alpha \cdot x^{j} R_{i} \beta\right) s_{j}} \\
& {[h \cdot \alpha,[a \cdot \beta, a \cdot \gamma]]=-a \cdot \alpha(\beta \cdot y \gamma)} \\
& {[h \cdot \alpha,[a \cdot \beta, k]]=0 .}
\end{aligned}
$$

Collecting all terms we find

$$
<h \cdot \alpha, a \cdot \beta>=\frac{1}{k+2}\left(3(\alpha \cdot y \beta)-\alpha \cdot x^{l} R_{l} \beta+\beta \cdot x^{l} R_{l} \alpha\right),
$$

and finally using the completeness relation

$$
<h \cdot \alpha, a \cdot \beta>=2(\alpha \cdot y \beta) .
$$




\subsection{The Involutive Automorphism}

As noted above the two involutive automorphisms $\tau$ and $\tau^{\prime}$ coincide when restricted to the subalgebra $\bar{g} \oplus \operatorname{sl}(2)$, whereas $\tau(X)=-\tau^{\prime}(X)$ for $X=$ $h \cdot \alpha+a \cdot \beta$. The action of $\tau$ on the subalgebra $s l(2)$ is given above, the action on $\bar{g}$ can be expressed by a matrix $T=\left(T^{i}{ }_{j}\right)$

$$
\tau\left(s_{i}\right)=s_{j} T^{j}{ }_{i}, \quad \text { with } \quad T_{j}^{i} T_{k}^{j}=\delta_{k}^{i} .
$$

From the commutators $\left[d, h_{i}\right]=\frac{1}{2} h_{i}$ and $\left[d, a_{i}\right]=-\frac{1}{2} a_{i}$ together with $\tau(d)=$ $-d$ we can deduce

$$
\tau(h \cdot \alpha)=a \cdot Z \alpha, \quad \tau(a \cdot \alpha)=h \cdot Z^{-1} \alpha,
$$

with some nonsingular $2 k \times 2 k$ matrix $Z$. The properties of this matrix $Z$ can be determined by consistency requirements:

$$
\begin{aligned}
& \tau([e, a \cdot \alpha])=[\tau(e), \tau(a \cdot \alpha)] \\
& \tau([k, h \cdot \alpha])=[\tau(k), \tau(h \cdot \alpha)] \\
& \Rightarrow Z^{-1}=-Z \\
& \tau\left(\left[s_{i}, a \cdot \alpha\right]\right)=\left[\tau\left(s_{i}\right), \tau(a \cdot \alpha)\right] \\
& \tau\left(\left[s_{i}, h \cdot \alpha\right]\right)=\left[\tau\left(s_{i}\right), \tau(h \cdot \alpha)\right] \\
& \Rightarrow Z R_{i} Z=-\tau\left(R_{i}\right) \equiv-R_{j} T^{j}{ }_{i}, \\
& \tau([h \cdot \alpha, a \cdot \beta])=[\tau(h \cdot \alpha), \tau(a \cdot \beta)] \quad \Rightarrow Z^{T} y Z=y, \quad Z^{T} x^{k} Z=T^{k}{ }_{l} x^{l} .
\end{aligned}
$$

Note that the symmetric matrix $y Z$ is positive definite, since the scalar product $\left\langle X, \tau^{\prime}(X)\right\rangle$ is negative definite. Changing the basis of the representation space for $\bar{\rho}$ yields modified matrices $R_{i}, Z, y$, and $x^{l}$

$$
R_{i} \mapsto S^{-1} R_{i} S, \quad Z \mapsto S^{-1} Z S, \quad y \mapsto S^{T} y S, \quad x^{l} \mapsto S^{T} x^{l} S .
$$

We choose a basis such that $y Z=c \mathbf{1}_{2 k}$, where $\mathbf{1}_{n}$ is the $n$-dimensional unit matrix, and $c$ is the positive constant introduced in Chapter 2, with the consequence

$$
\tau\left(R_{i}\right)=-R_{i}^{T}
$$

The matrix $Z$ is then antisymmetric, i.e. antihermitian and has therefore $k$ pairs of complex conjugate eigenvectors with eigenvalues $\pm i$. This allows us to further specifiy the basis such that

$$
Z=\left(\begin{array}{cc}
0 & -\eta^{T} \\
\eta & 0
\end{array}\right), \quad y=c\left(\begin{array}{cc}
0 & \eta^{T} \\
-\eta & 0
\end{array}\right)=-c \bar{Y}^{-1}, \quad \eta^{T} \eta=\mathbf{1}_{k},
$$

and to identify the Lie algebra elements $k, a \cdot \alpha, d$, and $s_{i}$ as generators of infinitesimal twist gauge, electromagnetic gauge, scale, and $\bar{G}$ transformations respectively. We will occasionally decompose the $2 k$-dimensional vectors $A$, $a, h, \alpha, \ldots$ into $k$-dimensional 'electric' and 'magnetic' parts $A_{(e)}, a_{(e)}, \ldots$ and $A_{(m)}, a_{(m)}, \ldots$ respectively (with $A_{(e)}=B$ and $\left.A_{(m)}=C\right)$. 


\section{Parametrization of the $\sigma$-Model}

\subsection{The General Structure}

We use an 'Iwasawa type' (KAN) decomposition of the Lie Algebra $g$ with the two subgroups $H=\exp K$ and $T=\exp A \exp N$ and coset representatives

$$
\pi: G / H \mapsto T,
$$

('triangular gauge'). The action of the group $G$ on these coset representatives is

$$
G \ni u: \pi(x) \mapsto v(x) \pi(x) u^{-1}, \quad \text { with } \quad v(x) \in H .
$$

The transformation with the constant parameter $u \in G$ is combined with a gauge transformation with parameter $v(x) \in H$ chosen such that the transformed coset representative is again an element of the triangular subgroup $T$. The infinitesimal form of these transformations is

$$
g \ni \delta g: \delta \pi(x)=\delta h(x) \pi(x)-\pi(x) \delta g, \quad \text { with } \quad \delta h(x) \in h .
$$

It is convenient to rewrite the variation of $\pi(x)$ in the form

$$
\delta \pi(x)=\left(\delta h(x)-\pi(x) \delta g \pi^{-1}(x)\right) \pi(x),
$$

that allows to determine $\delta h(x) \equiv \delta h(\pi(x), \delta g)$. Note that the Iwasawa decomposition and the triangular subgroup $T$ are very convenient but are not really needed; all we need are unique coset representatives $\pi$ that allow us to compute $\delta h(\pi(x), \delta g)$. For some of the coset spaces $\bar{G} / \bar{H}$ discussed in Appendix A we will actually not use the triangular subgroup.

Given $\pi(x)$ we can compute two Lie algebra valued 1-forms $\mathcal{A}$ and $\mathcal{J}$ $d \pi \pi^{-1}=\mathcal{A}+\mathcal{J}=\left(\mathcal{A}_{m}+\mathcal{J}_{m}\right) d x^{m}, \quad$ where $\quad \tau(\mathcal{A})=\mathcal{A}, \quad \tau(\mathcal{J})=-\mathcal{J}$, with transformation laws

$$
\delta \mathcal{J}=[\delta h, \mathcal{J}], \quad \delta \mathcal{A}=d \delta h+[\delta h, \mathcal{J}],
$$

i.e., $\mathcal{J}$ is a covariant ('matter') field and $\mathcal{A}$ can be interpreted as gauge connection. The $\sigma$-model Lagrangian is

$$
L=-\frac{1}{4}\langle\mathcal{J}, \mathcal{J}\rangle,
$$

with the implied contraction of 1-forms $\mathcal{J}_{m} d x^{m} \mathcal{J}_{n} d x^{n} \mapsto \mathcal{J}_{m} \mathcal{J}^{m}$. We can rewrite the resulting field equations

$$
\mathcal{D}_{m} \mathcal{J}^{m} \equiv \nabla_{m} \mathcal{J}^{m}-\left[\mathcal{A}_{m}, \mathcal{J}^{m}\right]=0,
$$


in the form

$$
\left.\mathcal{D}_{m} \mathcal{J}^{m}=\pi \nabla_{m} J^{m} \pi^{-1}, \quad \text { with } \quad J=\pi^{-1} \mathcal{J} \pi, \quad L=-\frac{1}{4}<J, J\right\rangle,
$$

and express $J$ in terms of $\pi$ as

$$
J=\frac{1}{2} \mu^{-1} d \mu, \quad \text { with } \quad \mu=\tau\left(\pi^{-1}\right) \pi,
$$

and with the linear transformation laws

$$
G \ni u: \mu \mapsto \tau(u) \mu u^{-1}, \quad J \mapsto u J u^{-1} .
$$

\subsection{The $S L(2) / S O(2) \sigma$-Model}

Dimensional reduction of gravity from 4 to 3 dimensions yields the well known $S L(2) / S O(2) \sigma$-model with two scalar fields, the square $\Delta$ of the Killing vector and the twist potential $\psi$.

The coset representatives $\tilde{\pi} \in \tilde{G} \equiv S L(2)$ are

$$
\tilde{\pi}=e^{\ln \Delta d} e^{\psi k} \text {. }
$$

We will need the expressions

$$
\delta \tilde{\pi} \tilde{\pi}^{-1}=\frac{\delta \Delta}{\Delta} d+\frac{\delta \psi}{\Delta} k, \quad \tilde{\pi}^{-1} \delta \tilde{\pi}=\frac{\delta \Delta}{\Delta} d+\left(\delta \psi-\psi \frac{\delta \Delta}{\Delta}\right) k,
$$

in order to compute the variation of the fields $\Delta$ and $\psi$ under infinitesimal transformations generated by $e$ (Ehlers transformation), $d$ (scale transformation), and $k$ (twist gauge transformation). The expression $\tilde{\pi} \delta \tilde{g}$ has already the required form for $\delta \tilde{g}=k$ or $d$ (the generators of the triangular subgroup $T$ ) and therefore the 'compensating' gauge transformation vanishes in these two cases:

$$
\begin{array}{llll}
\delta \tilde{g}=k: & \tilde{\pi}^{-1} \delta \tilde{\pi}=-k & \Rightarrow \delta \Delta=0, & \delta \psi=-1, \\
\delta \tilde{g}=d: & \tilde{\pi}^{-1} \delta \tilde{\pi}=-d \quad \Rightarrow \delta \Delta=-\Delta, & \delta \psi=-\psi .
\end{array}
$$

For the Ehlers transformation we find

$$
\delta \tilde{g}=e: \quad \delta \tilde{\pi} \tilde{\pi}^{-1}=\delta \tilde{h}-\tilde{\pi} e \tilde{\pi}^{-1}=\delta \tilde{h}-\Delta e+2 \psi d+\frac{\psi^{2}}{\Delta} k .
$$

Since the coefficient of $e$ in $\delta \tilde{\pi} \tilde{\pi}^{-1}$ has to vanish we must choose $\delta \tilde{h}=$ $\Delta(e-k)$ and obtain

$$
\delta \tilde{g}=e: \quad \delta \tilde{\pi} \tilde{\pi}^{-1}=2 \psi d+\left(\frac{\psi^{2}}{\Delta}-\Delta\right) k \Rightarrow \delta \Delta=2 \psi \Delta, \quad \delta \psi=\psi^{2}-\Delta^{2} .
$$


The commutators of these variations of the fields $\Delta$ and $\psi$ reflect the structure of the Lie algebra

$$
[\delta(X), \delta(Y)]=\delta([Y, X])
$$

where $\delta(X)$ are the variations under the transformation generated by $X \in \tilde{g}$.

The decomposition of $d \tilde{\pi} \tilde{\pi}^{-1}$ yields

$$
\tilde{\mathcal{A}}=\frac{d \psi}{2 \Delta}(k-e), \quad \tilde{\mathcal{J}}=\frac{d \Delta}{\Delta} d+\frac{d \psi}{2 \Delta}(e+k), \quad L=-\frac{(\partial \Delta)^{2}+(\partial \psi)^{2}}{4 \Delta^{2}}
$$

and the gauge invariant current is

$$
\tilde{J}=\tilde{\pi}^{-1} \tilde{\mathcal{J}} \tilde{\pi}=\frac{d \psi}{2 \Delta^{2}} e+\frac{\Delta d \Delta+\psi d \psi}{\Delta^{2}} d+\frac{\left(\Delta^{2}-\psi^{2}\right) d \psi-2 \psi \Delta d \Delta}{2 \Delta^{2}} k .
$$

We can easily translate these relations for Lie algebra and group elements into matrix equations, using the 2-dimensional matrix representation $\tilde{\rho}$

$$
\tilde{\rho}(\epsilon e+\delta d+\kappa k)=\left(\begin{array}{cc}
\frac{1}{2} \delta & \epsilon \\
\kappa & -\frac{1}{2} \delta
\end{array}\right), \quad<X, Y>=2 \operatorname{Tr}(\tilde{\rho}(X) \tilde{\rho}(Y))
$$

with

$$
\tilde{\rho}(\tilde{\tau}(X))=-\tilde{\rho}^{T}(X)=\tilde{y} \tilde{\rho}(X) \tilde{y}^{-1}, \quad \tilde{y}=i \sigma_{2}=\left(\begin{array}{cc}
0 & 1 \\
-1 & 0
\end{array}\right),
$$

and $\tilde{\rho}(\tilde{\pi})=\tilde{P}, \tilde{\rho}(\tilde{\mu})=\tilde{M}$,

$$
\tilde{P}=\left(\begin{array}{cc}
\sqrt{\Delta} & 0 \\
\frac{\psi}{\sqrt{\Delta}} & \frac{1}{\sqrt{\Delta}}
\end{array}\right), \quad \tilde{M}=\tilde{P}^{T} \tilde{P}=\left(\begin{array}{cc}
\Delta+\Delta^{-1} \psi^{2} & \Delta^{-1} \psi \\
\Delta^{-1} \psi & \Delta^{-1}
\end{array}\right) .
$$

\subsection{The $G / H \quad \sigma$-Model}

The fields of the $G / H$ (or $\left.G / H^{\prime}\right) \quad \sigma$-model are the scalars from the 4dimensional $\bar{G} / \bar{H} \sigma$-model described by coset representatives $\bar{\pi}$, the two scalars $\Delta$ and $\psi$ from 4 -dimensional gravity and $2 k$ scalar ('electromagnetic') potentials $A^{i}$ from the $k$ vector fields. Note that he twist vector $\omega_{m}$ now has a contribution from the electromagnetic potentials

$$
\omega=\omega_{m} d x^{m}=d \psi-\frac{1}{2} A \cdot y d A .
$$

We choose $G / H$ coset representatives $\pi$ in the triangular group $T \subset G$

$$
\pi=e^{\ln \Delta d} \bar{\pi} e^{a \cdot A+\psi k},
$$


with the $\bar{G} / \bar{H}$ coset representatives $\bar{\pi} \in \bar{T} \subset \bar{G}$. We will again need the expressions

$$
\begin{aligned}
\delta \pi \pi^{-1}= & \frac{\delta \Delta}{\Delta} d+\delta \bar{\pi} \bar{\pi}^{-1}+\frac{a \cdot \bar{P} \delta A}{\sqrt{\Delta}}+\frac{\delta \psi-\frac{1}{2} A \cdot y \delta A}{\Delta} k, \\
\pi^{-1} \delta \pi= & \frac{\delta \Delta}{\Delta} d+\bar{\pi}^{-1} \delta \bar{\pi}+a \cdot\left(\delta A+\bar{P}^{-1} \delta \bar{P} A-A \frac{\delta \Delta}{2 \Delta}\right) \\
& +\left(\delta \psi+\frac{1}{2} A \cdot y \delta A+\frac{1}{2} A \cdot y \bar{P}^{-1} \delta \bar{P} A-\psi \frac{\delta \Delta}{\Delta}\right) k,
\end{aligned}
$$

where $\bar{P}$ represents the group element $\bar{\pi} \in \bar{G}$ in the $2 k$-dimensional matrix representation $\bar{\rho}$

$$
\bar{g} \ni s_{i} \mapsto R\left(s_{i}\right)=R_{i}, \quad \bar{G} \ni \bar{\pi} \mapsto \bar{P}, \quad \text { with } \quad \bar{\pi} h \cdot \alpha \bar{\pi}^{-1}=h \cdot \bar{P} \alpha .
$$

For the infinitesimal transformations generated by $k$ (shift of the twist potential), $a$ (shift of the electromagnetic potentials), and $d$ (scale transformation) we have $\delta h=0$ and thus $\pi^{-1} \delta \pi=-\delta g$. The variations of the fields are:

$$
\begin{aligned}
& \delta g=k: \quad \delta \Delta=0, \quad \delta \bar{\pi}=0, \quad \delta A=0, \quad \delta \psi=-1, \\
& \delta g=a \cdot \alpha: \quad \delta \Delta=0, \quad \delta \bar{\pi}=0, \quad \delta A=-\alpha, \quad \delta \psi=\frac{1}{2} A \cdot y \alpha, \\
& \delta g=d: \quad \delta \Delta=-\Delta, \quad \delta \bar{\pi}=0, \quad \delta A=-\frac{1}{2} A, \quad \delta \psi=-\psi .
\end{aligned}
$$

For $\delta g=\delta \bar{g} \in \bar{g}$ we need the compensating gauge transformation $\delta h=$ $\delta \bar{h}(\bar{\pi}, \delta \bar{g})$ resulting in

$$
\begin{aligned}
\delta g=\delta \bar{g}: \quad \pi^{-1} \delta \pi=\pi^{-1} \delta \bar{h} \pi-\delta \bar{g} \\
\quad=\bar{\pi}^{-1} \delta \bar{h} \bar{\pi}-\delta \bar{g}+a \cdot R\left(\bar{\pi}^{-1} \delta \bar{h} \bar{\pi}\right) A+\frac{1}{2}\left(A \cdot y R\left(\bar{\pi}^{-1} \delta \bar{h} \bar{\pi}\right) A\right) k .
\end{aligned}
$$

Comparing coefficients and using $\bar{P}^{-1} \delta \bar{P}=R\left(\bar{\pi}^{-1} \delta \bar{\pi}\right)$ we find

$$
\delta \Delta=0, \quad \delta \bar{\pi}=\delta \bar{h} \bar{\pi}-\bar{\pi} \delta \bar{g}, \quad \delta A=R(\delta \bar{g}) A, \quad \delta \psi=0 .
$$

In order to determine the compensating gauge transformation $\delta h$ required for the infinitesimal transformations generated by $h$ (Harrison transformations) and $e$ (Ehlers transformation) we first compute $\pi \delta g \pi^{-1}$. For the Harrison transformations with $\delta g=h \cdot \alpha$ we obtain

$$
\begin{gathered}
\delta h-\pi \delta g \pi^{-1}=\delta h-\exp \left(\frac{a \cdot \bar{P} A}{\sqrt{\Delta}}+\frac{\psi}{\Delta} k\right) h \cdot \bar{P} \alpha \sqrt{\Delta} \exp -\left(\frac{a \cdot \bar{P} A}{\sqrt{\Delta}}+\frac{\psi}{\Delta} k\right) \\
=\delta h-h \cdot \bar{P} \alpha \sqrt{\Delta}-A \cdot y \alpha d+A \cdot x^{l} \alpha \bar{\pi} s_{l} \bar{\pi}^{-1} \\
\quad+\frac{1}{\sqrt{\Delta}} a \cdot \bar{P}\left(\alpha \psi-\frac{1}{4} A A \cdot y \alpha-\frac{1}{2} R_{l} A A \cdot x^{l} \alpha\right) \\
\quad-\frac{\psi}{\Delta} A \cdot y \alpha k+\frac{1}{6 \Delta} A \cdot y R_{l} A A \cdot x^{l} \alpha k .
\end{gathered}
$$

This requires

$$
\delta h(\pi, h \cdot \alpha)=(h \cdot \bar{P} \alpha+a \cdot Z \bar{P} \alpha) \sqrt{\Delta}-A \cdot x^{l} \alpha \delta \bar{h}\left(\bar{\pi}, s_{l}\right),
$$


and yields

$$
\begin{aligned}
\delta \pi \pi^{-1}= & -A \cdot y \alpha d-A \cdot x^{l} \alpha\left(\delta \bar{h}\left(\bar{\pi}, s_{l}\right)-\bar{\pi} s_{l} \bar{\pi}^{-1}\right)+a \cdot Z \bar{P} \alpha \sqrt{\Delta} \\
& +\frac{1}{\sqrt{\Delta}} a \cdot \bar{P}\left(\alpha \psi-\frac{1}{4} A A \cdot y \alpha-\frac{1}{2} R_{l} A A \cdot x^{l} \alpha\right) \\
& -\frac{\psi}{\Delta} A \cdot y \alpha k+\frac{1}{6 \Delta} A \cdot y R_{l} A A \cdot x^{l} \alpha k .
\end{aligned}
$$

Comparing coefficients we find the variations of the fields

$$
\begin{aligned}
& \delta \Delta=-A \cdot y \alpha \Delta, \\
& \delta \bar{\pi}=-A \cdot x^{l} \alpha\left(\delta \bar{h}\left(\bar{\pi}, s_{l}\right) \bar{\pi}-\bar{\pi} s_{l}\right), \\
& \delta A=\alpha \psi+c y^{-1} \bar{M} \alpha \Delta-\frac{1}{4} A A \cdot y \alpha-\frac{1}{2} R_{l} A A \cdot x^{l} \alpha, \\
& \delta \psi=-\frac{1}{2} A \cdot y \alpha \psi+\frac{c}{2} A \cdot \bar{M} \alpha \Delta-\frac{1}{12} A \cdot y R_{l} A A \cdot x^{l} \alpha,
\end{aligned}
$$

with the positive definite symmetric matrix $\bar{M}=\bar{P}^{T} \bar{P}$.

For the Ehlers transformations with $\delta g=e$ we obtain

$$
\begin{aligned}
\delta h-\pi \delta & g \pi^{-1}=\delta h-\exp \left(\frac{a \cdot \bar{P} A}{\sqrt{\Delta}}+\frac{\psi}{\Delta} k\right) \Delta e \exp -\left(\frac{a \cdot \bar{P} A}{\sqrt{\Delta}}+\frac{\psi}{\Delta} k\right) \\
=\delta & -e \Delta-h \cdot \bar{P} A \sqrt{\Delta}+2 \psi d+\frac{1}{2} A \cdot x^{l} A \bar{\pi} s_{l} \bar{\pi}^{-1} \\
& +\frac{1}{\sqrt{\Delta}} a \cdot \bar{P}\left(A \psi-\frac{1}{6} R_{l} A A \cdot x^{l} A\right) \\
& +\frac{\psi^{2}}{\Delta} k+\frac{1}{24 \Delta} A \cdot y R_{l} A A \cdot x^{l} A k .
\end{aligned}
$$

This requires

$$
\delta h(\pi, e)=\Delta(e-k)+(h \cdot \bar{P} A+a \cdot Z \bar{P} A) \sqrt{\Delta}-\frac{1}{2} A \cdot x^{l} A \delta \bar{h}\left(\bar{\pi}, s_{l}\right),
$$

and yields

$$
\begin{aligned}
\delta \pi \pi^{-1}= & 2 \psi d-\frac{1}{2} A \cdot x^{l} A\left(\delta \bar{h}\left(\bar{\pi}, s_{l}\right)-\bar{\pi} s_{l} \bar{\pi}^{-1}\right)+a \cdot Z \bar{P} A \sqrt{\Delta} \\
& +\frac{1}{\sqrt{\Delta}} a \cdot \bar{P}\left(A \psi-\frac{1}{6} R_{l} A A \cdot x^{l} A\right) \\
& +\frac{\psi^{2}-\Delta^{2}}{\Delta} k+\frac{1}{24 \Delta} A \cdot y R_{l} A A \cdot x^{l} A k
\end{aligned}
$$

Comparing coefficients we find the variations of the fields

$$
\begin{aligned}
& \delta \Delta=2 \psi \Delta \\
& \delta \bar{\pi}=-\frac{1}{2} A \cdot x^{l} A\left(\delta \bar{h}\left(\bar{\pi}, s_{l}\right) \bar{\pi}-\bar{\pi} s_{l}\right), \\
& \delta A=A \psi+c y^{-1} \bar{M} A \Delta-\frac{1}{6} R_{l} A A \cdot x^{l} A, \\
& \delta \psi=\psi^{2}-\Delta^{2}+\frac{c}{2} A \cdot \bar{M} A \Delta-\frac{1}{24} A \cdot y R_{l} A A \cdot x^{l} A .
\end{aligned}
$$

The decomposition of $d \pi \pi^{-1}$ yields

$$
\begin{aligned}
& \mathcal{A}=\overline{\mathcal{A}}+\frac{1}{2 \sqrt{\Delta}}(a \cdot \bar{P} d A-h \cdot Z \bar{P} d A)+\frac{\omega}{2 \Delta}(k-e), \\
& \mathcal{J}=\frac{d \Delta}{\Delta} d+\overline{\mathcal{J}}+\frac{1}{2 \sqrt{\Delta}}(a \cdot \bar{P} d A+h \cdot Z \bar{P} d A)+\frac{\omega}{2 \Delta}(e+k),
\end{aligned}
$$


where $\overline{\mathcal{A}}$ and $\overline{\mathcal{J}}$ are the quantities derived from $\bar{\pi}$. The Lagrangian is

$$
\left.L=-\frac{1}{4}\langle\mathcal{J}, \mathcal{J}\rangle=-\frac{1}{4}<\overline{\mathcal{J}}, \overline{\mathcal{J}}\right\rangle+\frac{c}{4 \Delta} \partial A \cdot \bar{M} \partial A-\frac{(\partial \Delta)^{2}+\omega^{2}}{4 \Delta^{2}} .
$$

The gauge invariant current is

$$
J=\pi^{-1} \mathcal{J} \pi=N^{-1} \hat{J} N, \quad N=\exp (a \cdot A+\psi k),
$$

with

$$
\hat{J}=\frac{\omega}{2 \Delta^{2}} e+\frac{1}{2 \Delta} h \cdot Z \bar{M} d A+\frac{d \Delta}{\Delta} d+\bar{J}+\frac{1}{2} a \cdot d A+\frac{\omega}{2} k .
$$

In order to compute $N^{-1} \hat{J} N$ let us first evaluate $N^{-1} X N$ for all $X \in g$

$$
\begin{aligned}
& N^{-1} e N=e-h \cdot A+2 \psi d-\frac{1}{2} A \cdot x^{i} A s_{i} \\
& -a \cdot A \psi-\frac{1}{6} a \cdot R_{i} A A \cdot x^{i} A-\psi^{2} k-\frac{1}{24} A \cdot x^{i} A A \cdot y R_{i} A k, \\
& N^{-1} h \cdot \alpha N=h \cdot \alpha-A \cdot y \alpha d+A \cdot x^{i} \alpha s_{i}+a \cdot \alpha \psi+\frac{1}{4} a \cdot A A \cdot y \alpha \\
& +\frac{1}{2} a \cdot R_{i} A A \cdot x^{i} \alpha+A \cdot y \alpha \psi k+\frac{1}{6} A \cdot x^{i} A A \cdot y R_{i} \alpha k \text {, } \\
& N^{-1} d N=d-\frac{1}{2} a \cdot A-\psi k \text {, } \\
& N^{-1} s_{i} N=s_{i}+a \cdot R_{i} A+\frac{1}{2} A \cdot y R_{i} A k, \\
& N^{-1} a \cdot \alpha N=a \cdot \alpha+A \cdot y \alpha k \text {, } \\
& N^{-1} k N=k \text {, }
\end{aligned}
$$

Expanding $J$ with respect to the generators

$$
J=\frac{1}{2} J^{e} e+\frac{1}{2} h \cdot Z J^{h}+J^{d} d+J^{i} s_{i}+\frac{1}{2} a \cdot J^{a}+\frac{1}{2} J^{k} k,
$$

and collecting all terms we obtain the conserved currents

$$
\begin{aligned}
J^{e}= & \frac{\omega}{\Delta^{2}} \\
J^{h}= & \frac{1}{\Delta} \bar{M} d A+Z A \frac{\omega}{\Delta^{2}} \\
J^{d}= & \frac{d \Delta}{\Delta}-\frac{c}{2 \Delta} A \cdot \bar{M} d A+\frac{\omega}{\Delta^{2}} \psi \\
J^{i} s_{i}= & \bar{J}+\frac{1}{2 \Delta} A \cdot x^{i} Z \bar{M} d A s_{i}-\frac{\omega}{4 \Delta^{2}} A \cdot x^{i} A s_{i}, \\
J^{a}= & d A-A \frac{d \Delta}{\Delta}+2 R(\bar{J}) A+\frac{1}{2 \Delta} R_{i} A A \cdot x^{i} Z \bar{M} d A+\frac{1}{\Delta} Z \bar{M} d A \psi \\
& +\frac{c}{4 \Delta} A A \cdot \bar{M} d A-\frac{\omega}{\Delta^{2}} A \psi-\frac{\omega}{6 \Delta^{2}} R_{i} A A \cdot x^{i} A, \\
J^{k}= & \omega+A \cdot y d A-2 \frac{d \Delta}{\Delta} \psi+A \cdot y R(\bar{J}) A+\frac{c}{\Delta} A \cdot \bar{M} d A \psi \\
& -\frac{c}{6 \Delta} A \cdot x^{i} A A \cdot R_{i}^{T} \bar{M} d A-\frac{\omega}{\Delta^{2}} \psi^{2}-\frac{\omega}{24 \Delta^{2}} A \cdot x^{i} A A \cdot y R_{i} A,
\end{aligned}
$$


and the Lagrangian

$$
L=-\frac{1}{4}<J, J>=-\frac{1}{4}\left(J^{i} J^{j}<s_{i}, s_{j}>-c J^{a} \cdot J^{h}+J^{d} J^{d}+J^{e} J^{k}\right) .
$$

Using the identity $x^{i}<s_{i}, s_{j}>=-2 y R_{j}$ it is straight forward, although tedious, to verify that this reproduces the expression given above.

\section{Application to Black Holes}

In our previous paper with Gibbons [1] we have used the $\sigma$-model formulation in order to generalize a number of results on stationary black holes of the Einstein-Maxwell theory to a large class of 4-dimensional theories with abelian gauge fields and scalars. With the more detailed knowledge of their group theoretical structure gained in this paper, some further applications are conceivable. Here we just give simple derivations of two such results for strictly stationary single black holes. First we generalize a quadratic mass formula of Heusler [沟 to all the theories under consideration. It is quite remarkable, how the use of the group theoretical structure simplifies the derivation. As a second application we state the action of the EhlersHarrison transformation on the charges of the black hole.

Consider a strictly stationary single black hole solution for one of the theories discussed in this article in its dimensionally reduced form. We can choose suitable coordinates on $\Sigma_{3}$ as well as a suitable gauge such that the behaviour of the fields at infinity is

$$
h_{m n}(x)=\delta_{m n}+O\left(\frac{1}{r}\right), \quad \pi(x)=\exp \left(\frac{\pi_{1}}{r}+O\left(\frac{1}{r^{2}}\right)\right) .
$$

The element $\pi_{1} \in t$ (where $t$ is the Lie algebra of the triangular group $T$ ) contains the charges

$$
\pi_{1}=-2 m d+2 n k+a \cdot q+\bar{\pi}_{1},
$$

the total mass $m$, the NUT-charge $n$, the combined electric and magnetic charges $q$, and scalar charges determined by $\bar{\pi}_{1} \in \bar{t}$.

Integrating the conserved currents $J$ over a closed surface $\Sigma$ enclosing the black hole yields the Lie algebra valued charge

$$
Q=\frac{1}{4 \pi} \oint J^{m} d \Sigma_{m}
$$


Evaluating this integral at infinity we obtain

$$
Q=\frac{1}{2}\left(\tau\left(\pi_{1}\right)-\pi_{1}\right)=2 m d-n(e+k)-\frac{1}{2}(a \cdot q+h \cdot Z q)+Q^{i} s_{i},
$$

with the scalar charges $\bar{Q}=Q^{i} s_{i}=\frac{1}{2}\left(\tau\left(\bar{\pi}_{1}\right)-\bar{\pi}_{1}\right)$.

Due to the assumption of strict stationarity, the field $\Delta$ vanishes on the horizon $\mathcal{H}$ and is positive everywhere outside $\mathcal{H}$. The boundary conditions at the horizon [7] imply that $\psi, A$, and the surface gravity $\kappa=\frac{1}{2} \sqrt{h^{m n} \partial_{m} \Delta \partial_{n} \Delta}$ are constant on the horizon, whereas the scalars fields $\phi^{i}$ are finite but may vary. Moreover, the twist vector $\omega_{m}$ vanishes linearly with $\Delta$, and the area of the horizon is given by

$$
a_{\mathcal{H}}=\lim _{\Delta \rightarrow 0} \frac{1}{\Delta} \oint_{\Delta=\text { const. }} n^{m} d \Sigma_{m},
$$

where $n$ is the unit normal to the surface $\Delta=$ const. with respect to the rescaled metric $h_{m n}$.

\subsection{A Quadratic Mass Formula}

Evaluating the integral for $Q$ on the horizion yields

$$
Q=N_{\mathcal{H}}^{-1} Q_{\mathcal{H}} N_{\mathcal{H}}
$$

where $N_{\mathcal{H}}$ is the value of $N=\exp (a \cdot A+\psi k)$ on the horizon, and the charges $Q_{\mathcal{H}}$ on the horizon are

$$
Q_{\mathcal{H}}=\frac{1}{4 \pi} \oint_{\mathcal{H}} \hat{J}^{m} d \Sigma_{m}=2 m_{\mathcal{H}} d-n_{\mathcal{H}} e-\frac{1}{2} h \cdot Z q_{\mathcal{H}},
$$

with the 'irreducible mass'

$$
m_{\mathcal{H}}=\frac{1}{8 \pi} \oint_{\mathcal{H}} \frac{\partial_{m} \Delta}{\Delta} d \Sigma^{m}=\frac{1}{4 \pi} \kappa_{\mathcal{H}} a_{\mathcal{H}}
$$

and the NUT and vector charges 'at the horizon'

$$
n_{\mathcal{H}}=-\frac{1}{8 \pi} \oint_{\mathcal{H}} \frac{\omega_{m}}{\Delta^{2}} d \Sigma^{m}, \quad q_{\mathcal{H}}=-\frac{1}{4 \pi} \oint_{\mathcal{H}} \frac{\bar{M} \partial_{m} A}{\Delta} d \Sigma^{m}
$$

Note that $Q_{\mathcal{H}}$ has no contributions with the generators $s_{i}$, a, and $k$, because the corresponding terms in $\hat{J}$ carry no inverse powers of $\Delta$.

Using the invariance of the scalar product, the relation between $Q$ and $Q_{\mathcal{H}}$ implies $\left\langle Q, Q>=<Q_{\mathcal{H}}, Q_{\mathcal{H}}>\right.$. Furthermore the structure of the 
scalar product (pairing $e$ with $k$, etc.) yields $\left\langle Q_{\mathcal{H}}, Q_{\mathcal{H}}\right\rangle=4 m_{\mathcal{H}}^{2}$ and hence the desired quadratic mass formula

$$
m^{2}+n^{2}-\frac{c}{4} q^{T} q+<\bar{Q}, \bar{Q}>_{\bar{g}}=m_{\mathcal{H}}^{2},
$$

extending the well known relation $m^{2}+n^{2}-q^{2}-p^{2}=m_{\mathcal{H}}^{2}$ for the generalized Reissner-Nordstrøm solutions with electric and magnetic charges $q$ and $p$ to a large class of theories.

\subsection{The Action of $G$ on Black Holes}

The action of $G$, given explicitly in Section 4.3 for the infinitesimal transformations, preserves the strict stationarity as well as the boundary conditions on the horizon, and leaves the irreducible mass invariant. The action of $G$ on the conserved currents is

$$
G \ni u: J \mapsto u J u^{-1}
$$

In order to preserve the boundary conditions at infinity, we have to restrict the transformations to the subgroup $H$, with the action on the Lie algebra valued charge $Q$ given by

$$
H \ni u: Q \mapsto u Q u^{-1}
$$

For the infinital transformation generated by $\delta g=e-k$ this yields

$$
\delta m=-2 n, \quad \delta n=2 m, \quad \delta q=Z q, \quad \delta \bar{Q}=0,
$$

for the transformation generated by $\delta g=h \cdot \alpha+a \cdot Z \alpha$

$$
\begin{array}{ll}
\delta m=-\frac{c}{2} q \cdot Z \alpha, & \delta q=2 \alpha n-2 Z \alpha m+2 R(\bar{Q}) Z \alpha, \\
\delta n=\frac{c}{2} q \cdot \alpha, & \delta \bar{Q}=-\frac{1}{2} q \cdot x^{l} \alpha\left(s_{l}-\tau\left(s_{l}\right)\right),
\end{array}
$$

and for the transformations generated by $\delta g=\delta \bar{g} \in \bar{h}$

$$
\delta m=0, \quad \delta n=0, \quad \delta q=R(\delta \bar{g}) q, \quad \delta \bar{Q}=[\delta \bar{g}, \bar{Q}] .
$$

Starting from a nondegenerate black hole (i.e. $m_{\mathcal{H}}>0$ ) with charges $m, n, q$, and $\bar{Q}$, we can apply a suitable group element from $H$ to obtain a new black hole with the same $m_{\mathcal{H}}$, and with charges $n=q=0$. Using the identity $Q=N_{\mathcal{H}}^{-1} Q_{\mathcal{H}} N_{\mathcal{H}}$ we conclude $n_{\mathcal{H}}=q_{\mathcal{H}}=0$, and therefore $m=m_{\mathcal{H}}$, $\bar{Q}=\psi_{\mathcal{H}}=A_{\mathcal{H}}=0$. 


\section{A Appendix: The Individual Models}

Dimensional reduction of 4-dimensional theories consisting of a $\bar{G} / \bar{H} \sigma$ model coupled to $k$ abelian vector fields and gravity to 3 dimensions yields $(\operatorname{dim} \bar{G}-\operatorname{dim} \bar{H})+2 k+2$ scalars coupled to gravity. The conditions that all these scalars form one $G / H \sigma$-model have been discussed in [1]. In Table 1, reproduced from that paper, we list 15 different possibilities, all with a simple Lie group $G$. In the following we discuss some of these cases in detail and finally indicate a general procedure applicable to all cases.

\section{A.1 $S L(n+2) / S O(n, 2)$}

These are the Kałuza-Klein theories obtained from pure gravity in $D=n+4$ dimensions by dimensional reduction with respect to $n$ commuting space like Killing vectors. The 4-dimensional theory consists of $k=n$ vector fields and a $G L(n) / S O(n) \sigma$-model with $\frac{1}{2} n(n+1)$ scalars. Let $\hat{\pi}$ be the $G L(n) / S O(n)$ coset representative and $\hat{\rho}$ the $k$-dimensional matrix representation

$$
\hat{\rho}: \hat{\pi} \mapsto \hat{\rho}(\hat{\pi})=\hat{P}, \quad \tau(\hat{P})=\hat{P}^{T-1}, \quad \hat{\rho}\left(s_{i}\right)=r_{i} .
$$

The action for the 4-dimensional theory is given by

$$
<\bar{J}, \bar{J}>_{\bar{g}}=2 \operatorname{Tr}(\hat{J} \hat{J})-\frac{2}{n+2} \operatorname{Tr}(\hat{J}) \operatorname{Tr}(\hat{J}), \quad c=1, \quad \tilde{\mu}=\hat{M}, \quad \tilde{\nu}=0,
$$

where the symmetric matrix $\hat{M}=\hat{P}^{T} \hat{P}$ contains the (rescaled) scalar products of the $n$ Killing vectors, and the $\sigma$-model currents are

$$
\hat{\rho}(\bar{J})=\hat{J}=\frac{1}{2} \hat{M}^{-1} d \hat{M} .
$$

The theory is invariant under the action of $G L(n)$ and $S O(n)$

$$
G L(n) \ni u: B_{a} \mapsto u B_{a}, \quad \hat{P} \mapsto v \hat{P} u^{-1}, \quad \text { with } \quad v \in S O(n) .
$$

Further dimensional reduction with respect to a time like Killing vector yields (with a suitable choice for $\eta$ ) the 3-dimensional theory with

$$
\bar{M}=\left(\begin{array}{cc}
\hat{M} & 0 \\
0 & \hat{M}^{-1}
\end{array}\right), \quad R_{i}=\left(\begin{array}{cc}
r_{i} & 0 \\
0 & -r_{i}^{T}
\end{array}\right), \quad y=\left(\begin{array}{cc}
0 & \mathbf{1}_{n} \\
-\mathbf{1}_{n} & 0
\end{array}\right) .
$$

This model has the unique property that the scalar potentials $A$ transform under a reducible representation of the group $\bar{G}=G L(n)$, due to the fact 
that the action of $\bar{G}$ on the field strengths $B_{a b}$ involves no duality transformations; this leads to an unambiguous decomposition into electric and magnetic potentials $A_{(e)}$ and $A_{(m)}$ respectively.

In order to exhibit the $S L(n+2) / S O(n, 2) \sigma$-model consider the $(n+2)$ dimensional matrix representation $\rho(X)$ of the element $X=\epsilon e+h \cdot \alpha+\delta d+$ $\lambda^{i} s_{i}+a \cdot \beta+\kappa k$ of the Lie algebra $\operatorname{sl}(n+2)$ and the involutive automorphism $\rho(\tau(X))=-D^{-1} \rho^{T}(X) D$ with

$\rho(X)=\left(\begin{array}{ccc}\frac{1}{2} \delta & -\alpha_{(m)}^{T} & \epsilon \\ \beta_{(e)} & \lambda^{i} r_{i} & \alpha_{(e)} \\ \kappa & \beta_{(m)}^{T} & -\frac{1}{2} \delta\end{array}\right)-\frac{\operatorname{Tr}\left(\lambda^{i} r_{i}\right)}{n+2} \mathbf{1}_{n+2}, \quad D=\left(\begin{array}{ccc}1 & 0 & 0 \\ 0 & -\mathbf{1}_{n} & 0 \\ 0 & 0 & 1\end{array}\right)$,

and with the scalar product $\langle X, Y\rangle=2 \operatorname{Tr}(\rho(X) \rho(Y))$. Finally we obtain

$$
P=\rho(\pi)=(\operatorname{det} \hat{P})^{-\frac{1}{n+2}}\left(\begin{array}{ccc}
\sqrt{\Delta} & 0 & 0 \\
\hat{P} A_{(e)} & \hat{P} & 0 \\
\frac{1}{\sqrt{\Delta}}\left(\psi+\frac{1}{2} A_{(m)}^{T} A_{(e)}\right) & \frac{1}{\sqrt{\Delta}} A_{(m)}^{T} & \frac{1}{\sqrt{\Delta}}
\end{array}\right),
$$

and $\rho(J)=\frac{1}{2} M^{-1} d M$ with $M=P^{T} D P$.

\section{A.2 $S U(p+1, q+1) / S(U(p, 1) \times U(1, q))$}

These are 4-dimensional theories with $k=p+q$ vector fields and the $2 p q$ real scalars from $U(p, q) /(U(p) \times U(q))$. For $q=0$ there are no scalars, the only effect of the $U(p) / U(p) \sigma$-model is the action of $U(p)$ on the field strength. For $p=1, q=0$ this is the Einstein-Maxwell theory; the theories with $p>1, q=0$ are generalizations with several vector fields.

Let $\bar{\pi}$ be the $U(p, q) /(U(p) \times U(q))$ coset representative, $\hat{\rho}$ the $k$-dimensional complex matrix representation

$$
\hat{\rho}: \bar{\pi} \mapsto \hat{\rho}(\bar{\pi})=\hat{P}, \quad \hat{V} \hat{P}=\left(\hat{P}^{+}\right)^{-1} \hat{V}, \quad \tau(\hat{P})=\left(\hat{P}^{+}\right)^{-1},
$$

with the real $U(p, q)$ metric $\hat{V}=\hat{V}^{T}=\hat{V}^{*}=\hat{V}^{-1}$, and $\bar{\rho}$ the corresponding $2 k$-dimensional real representation

$$
\bar{\rho}: \bar{\pi} \mapsto \bar{\rho}(\bar{\pi})=\bar{P}=\left(\begin{array}{rr}
\operatorname{Re} \hat{P} & -\operatorname{Im} \hat{P} \\
\operatorname{Im} \hat{P} & \operatorname{Re} \hat{P}
\end{array}\right) .
$$

Decomposing the hermitian matrix $\hat{M}=\hat{P}^{+} \hat{P}$ satisfying $\hat{M} \hat{V} \hat{M}=\hat{V}$ into real and imaginary parts $\tilde{M}=\tilde{M}^{T}=\operatorname{Re} \hat{M}$ and $\tilde{N}=-\tilde{N}^{T}=\operatorname{Im} \hat{M}$ we obtain the relations

$$
\tilde{M} \hat{V} \tilde{M}-\tilde{N} \hat{V} \tilde{N}=\hat{V}, \quad \tilde{M} \hat{V} \tilde{N}+\tilde{N} \hat{V} \tilde{M}=0
$$


This allows us to express the action for the 4-dimensional theory with $c=4$ in terms of the symmetric matrices

$$
\tilde{\mu}=\hat{V} \tilde{M}^{-1} \hat{V}, \quad \tilde{\nu}=\hat{V} \tilde{M}^{-1} \tilde{N}
$$

and the invariant scalar product on $u(p, q)$

$$
<X, Y>_{\bar{g}}=2 \operatorname{Tr}(\hat{\rho}(X) \hat{\rho}(Y))-\frac{2}{p+q+2} \operatorname{Tr} \hat{\rho}(X) \operatorname{Tr} \hat{\rho}(Y) .
$$

Note, however, that $\operatorname{det} \hat{M}=1$ and therfore $\operatorname{Tr} \hat{\rho}(J)=0$.

Choosing $\eta=\hat{V}$, we obtain the the 3-dimensional theory with

$$
\bar{M}=\left(\begin{array}{cc}
\tilde{M} & -\tilde{N} \\
\tilde{N} & \tilde{M}
\end{array}\right), \quad y=c\left(\begin{array}{cc}
0 & \hat{V} \\
-\hat{V} & 0
\end{array}\right) .
$$

In order to construct the $(k+2)$-dimensional matrix representation $\rho$ of $S U(p+1, q+1)$ we first rearrange the $2 k$ real components of $A$ into a $k$ dimensional complex vector $\mathbf{A}=A_{(e)}+i A_{(m)}$ and similarly for $a, h, \alpha$, etc. The element $X=\epsilon e+h \cdot \alpha+\delta d+\lambda^{i} s_{i}+a \cdot \beta+\kappa k$ of $s u(p+1, q+1)$ is represented by

$$
\rho(X)=\left(\begin{array}{ccc}
\frac{1}{2} \delta & -\sqrt{2} \boldsymbol{\alpha}^{+} \hat{V} & \epsilon \\
i \sqrt{2} \boldsymbol{\beta} & \lambda^{i} \hat{\rho}\left(s_{i}\right) & i \sqrt{2} \boldsymbol{\alpha} \\
\kappa & \sqrt{2} \boldsymbol{\beta}^{+} \hat{V} & -\frac{1}{2} \delta
\end{array}\right)-\frac{\operatorname{Tr}\left(\lambda^{i} \hat{\rho}\left(s_{i}\right)\right)}{k+2} \mathbf{1}_{k+2}
$$

such that $\rho(X)=-V^{-1} \rho^{+}(X) V$ and $\rho(\tau(X))=-D^{-1} \rho^{+}(X) D$ with

$$
V=\left(\begin{array}{ccc}
0 & 0 & -i \\
0 & \hat{V} & 0 \\
i & 0 & 0
\end{array}\right), \quad D=\left(\begin{array}{ccc}
1 & 0 & 0 \\
0 & -\mathbf{1}_{k} & 0 \\
0 & 0 & 1
\end{array}\right)
$$

and with the scalar product $\langle X, Y\rangle=2 \operatorname{Tr}(\rho(X) \rho(Y))$. Finally we obtain

$$
P=\rho(\pi)=(\operatorname{det} \hat{P})^{-\frac{1}{k+2}}\left(\begin{array}{ccc}
\sqrt{\Delta} & 0 & 0 \\
i \sqrt{2} \hat{P} \mathbf{A} & \hat{P} & 0 \\
\frac{1}{\sqrt{\Delta}}\left(\psi+i \mathbf{A}^{+} \hat{V} \mathbf{A}\right) & \frac{\sqrt{2}}{\sqrt{\Delta}} \mathbf{A}^{+} \hat{V} & \frac{1}{\sqrt{\Delta}}
\end{array}\right)
$$

the twist vector $\omega=d \psi-2 \operatorname{Im} \mathbf{A}^{+} \hat{V} d \mathbf{A}$, and $\rho(J)=\frac{1}{2} M^{-1} d M$ with $M=$ $P^{+} D P$. 


\section{A.3 $S O(p+2, q+2) /(S O(p, 2) \times S O(2, q))$}

These are 4-dimensional theories with $k=p+q$ vector fields, with $p q$ scalars from $S O(p, q) /(S O(p) \times S O(q))$, and with a dilaton $\varphi$ and an axion $\chi$ from $S L(2) / S O(2)$. Some well known examples are the Einstein-Maxwell-dilatonaxion theory with $p=1, q=0$ [8], the bosonic sector of $N=4$ supergravity with $p=6, q=0$, and the (dimensionally reduced) bosonic sector of 10dimensional supergravity with $p=q=6$.

Let $\hat{\pi}$ be the $S O(p, q) /(S O(p) \times S O(q))$ coset representative and $\hat{\rho}$ the $k$-dimensional matrix representation

$$
\hat{\rho}: \hat{\pi} \mapsto \hat{\rho}(\hat{\pi})=\hat{P}, \quad \hat{V} \hat{P}=\hat{P}^{T-1} \hat{V}, \quad \tau(\hat{P})=\hat{P}^{T-1},
$$

with the real $S O(p, q)$ metric $\hat{V}=\hat{V}^{T}=\hat{V}^{-1}$, and consider the action for the 4-dimensional theory with

$$
<\bar{J}, \bar{J}>_{\bar{g}}=\frac{(\partial \varphi)^{2}+(\partial \chi)^{2}}{\varphi^{2}}+\operatorname{Tr}(\hat{J} \hat{J}), \quad c=1, \quad \tilde{\mu}=\varphi \hat{M}, \quad \tilde{\nu}=\chi \hat{V},
$$

where

$$
\hat{M}=\hat{P}^{T} \hat{P}, \quad \hat{J}=\frac{1}{2} \hat{M}^{-1} d \hat{M} .
$$

Choosing $\eta=\hat{V}$, we obtain the 3 -dimensional theory with $\bar{M}=\hat{M} \otimes \tilde{M}$, $y=\hat{V} \otimes \tilde{y}, \tilde{y}=i \sigma_{2}$, where

$$
\tilde{P}=\left(\begin{array}{cc}
\sqrt{\varphi} & 0 \\
\frac{\chi}{\sqrt{\varphi}} & \frac{1}{\sqrt{\varphi}}
\end{array}\right), \quad \tilde{M}=\tilde{P}^{T} \tilde{P}=\left(\begin{array}{cc}
\varphi+\varphi^{-1} \chi^{2} & \varphi^{-1} \chi \\
\varphi^{-1} \chi & \varphi^{-1}
\end{array}\right)
$$

parametrizes the $S L(2) / S O(2) \sigma$-model.

In order to construct the $(k+4)$-dimensional matrix representation $\rho$ of $S O(p+2, q+2)$ we first rearrange the $2 k$ components of $A$ into a $k \times 2$ matrix $\mathbf{A}=\left(A_{(e)}, A_{(m)}\right)$ and similarly for $a, h, \alpha, \ldots$ and split the generators $s_{i}$ of $\bar{G}$ into $\hat{s}_{i} \in \hat{g}=s o(p, q)$ and $\tilde{s}_{i} \in \tilde{g}=s l(2)$. The element $X=$ $\epsilon e+h \cdot \alpha+\delta d+\lambda^{i} s_{i}+a \cdot \beta+\kappa k$ of $s o(p+2, q+2)$ is represented by

$$
\rho(X)=\left(\begin{array}{ccc}
\frac{1}{2} \delta \mathbf{1}_{2}+\tilde{\lambda}^{i} \tilde{\rho}\left(\tilde{s}_{i}\right) & -\boldsymbol{\alpha}^{T} \hat{V} & \epsilon \mathbf{1}_{k} \\
\boldsymbol{\beta} \tilde{y} & \hat{\lambda}^{i} \hat{\rho}\left(\hat{s}_{i}\right) & \boldsymbol{\alpha} \tilde{y} \\
\kappa \mathbf{1}_{2} & \boldsymbol{\beta}^{T} \hat{V} & -\frac{1}{2} \delta \mathbf{1}_{2}+\tilde{\lambda}^{i} \tilde{\rho}\left(\tilde{s}_{i}\right)
\end{array}\right),
$$

such that $\rho(X)=-V^{-1} \rho^{T}(X) V$ and $\rho(\tau(X))=-D^{-1} \rho^{T}(X) D$ with

$$
V=\left(\begin{array}{ccc}
0 & 0 & \tilde{y} \\
0 & \hat{V} & 0 \\
-\tilde{y} & 0 & 0
\end{array}\right), \quad D=\left(\begin{array}{ccc}
\mathbf{1}_{2} & 0 & 0 \\
0 & -\mathbf{1}_{k} & 0 \\
0 & 0 & \mathbf{1}_{2}
\end{array}\right)
$$


and with the scalar product $\langle X, Y\rangle=\operatorname{Tr}(\rho(X) \rho(Y))$. Finally we obtain

$$
P=\rho(\pi)=\left(\begin{array}{ccc}
\Delta^{1 / 2} \tilde{P} & 0 & 0 \\
\hat{P} \mathbf{A} \tilde{y} & \hat{P} & 0 \\
\Delta^{-1 / 2}\left(\psi \mathbf{1}_{2}+\frac{1}{2} \mathbf{A}^{T} \hat{V} \mathbf{A} \tilde{y}\right) & \Delta^{-1 / 2} \mathbf{A}^{T} \hat{V} & \Delta^{-1 / 2} \tilde{P}
\end{array}\right)
$$

and $\rho(J)=\frac{1}{2} M^{-1} d M$ with $M=P^{T} D P$.

\section{A.4 SO* $(2 n+4) / U(n, 2)$}

These are 4-dimensional theories with $k=2 n$ vector fields and the $n(n-1)$ scalars from $S O^{*}(2 n) / U(n) \times S U(2) / S U(2)$. In the following we assume $n>1$, since for $n=1$ this is the $S U(3,1) / U(2,1)$ theory already discussed in Section A.2. The $4 n$ electromagnetic potentials transform under the $2 n$ dimensional representation $\hat{\rho}$ of $S O^{*}(2 n)$ and under the 2-dimensional representation $\tilde{\rho}$ of $S U(2)$, and we will therefore split the generators $s_{i}$ of $\bar{G}$ into $\hat{s}_{i} \in \hat{g}=s o^{*}(2 n)$ and $\tilde{s}_{i} \in \tilde{g}=s u(2)$.

The group $S O^{*}(2 n)$ is defined as the subgroup of $S O(2 n ; \mathbf{C})$ that leaves the antihermitian form $\varphi^{+} i \sigma_{2} \otimes \mathbf{1}_{n} \chi$ invariant. Allowing for a change of basis we obtain

$$
\hat{\rho}: \hat{s}_{i} \mapsto \hat{R}_{i}, \quad \hat{V} \hat{R}_{i}+\hat{R}_{i}^{+} \hat{V}=0, \quad \hat{W} \hat{R}_{i}+\hat{R}_{i}^{T} \hat{W}=0, \quad \tau\left(\hat{R}_{i}\right)=-\hat{R}_{i}^{+},
$$

with matrices $\hat{V}=-\hat{V}^{+}$and $\hat{W}=\hat{W}^{T}$ such that $\hat{C} \equiv \hat{V}^{-1} \hat{W}^{+}=\hat{W}^{-1} \hat{V}^{T}$. This implies the reality condition $\hat{R}_{i}=\hat{C} \hat{R}_{i}^{*} \hat{C}^{-1}$ with $\hat{C} \hat{C}^{*}=-\mathbf{1}_{2 n}$. Hence the representation $\hat{\rho}$ is 'pseudo real', i.e., is equivalent to its complex conjugate but cannot be written with real matrices; the same holds true for the 2-dimensional representation $\tilde{\rho}$ of $s u(2)$ with

$$
\tilde{\rho}: \tilde{s}_{i} \mapsto \tilde{R}_{i}=\frac{i}{2} \sigma_{i}=\tau\left(\tilde{R}_{i}\right)=-\tilde{R}_{i}^{+}=-\tilde{y}^{-1} \tilde{R}_{i}^{T} \tilde{y}, \quad \tilde{y}=i \sigma_{2}=\left(\begin{array}{cc}
0 & 1 \\
-1 & 0
\end{array}\right)
$$

and $\tilde{R}_{i}=\tilde{y} \tilde{R}_{i}^{*} \tilde{y}^{-1}$.

We can now construct the $(2 n+4)$-dimensional matrix representation $\rho$ of $s o^{*}(2 n+4)$ with the Lie algebra element $X=\epsilon e+h \cdot \alpha+\delta d+\lambda^{i} s_{i}+a \cdot \beta+\kappa k$ represented by

$$
\rho(X)=\left(\begin{array}{ccc}
\frac{1}{2} \delta \mathbf{1}_{2}+\tilde{\lambda}^{i} \tilde{\rho}\left(\tilde{s}_{i}\right) & -\boldsymbol{\alpha}^{+} \hat{V} & \epsilon \mathbf{1}_{2} \\
\boldsymbol{\beta} & \hat{\lambda}^{i} \hat{\rho}\left(\hat{s}_{i}\right) & \boldsymbol{\alpha} \\
\kappa \mathbf{1}_{2} & \boldsymbol{\beta}^{+} \hat{V} & -\frac{1}{2} \delta \mathbf{1}_{2}+\tilde{\lambda}^{i} \tilde{\rho}\left(\tilde{s}_{i}\right)
\end{array}\right)
$$


such that $V \rho(X)+\rho^{+}(X) V=0, W \rho(X)+\rho^{T}(X) W=0$, and $\rho(\tau(X))=$ $-D^{-1} \rho^{+}(X) D$ with

$$
V=\left(\begin{array}{ccc}
0 & 0 & -\mathbf{1}_{2} \\
0 & \hat{V} & 0 \\
\mathbf{1}_{2} & 0 & 0
\end{array}\right), W=\left(\begin{array}{ccc}
0 & 0 & \tilde{y} \\
0 & \hat{W} & 0 \\
-\tilde{y} & 0 & 0
\end{array}\right), D=\left(\begin{array}{ccc}
\mathbf{1}_{2} & 0 & 0 \\
0 & -\mathbf{1}_{2 n} & 0 \\
0 & 0 & \mathbf{1}_{2}
\end{array}\right)
$$

and with the scalar product $\langle X, Y\rangle=\operatorname{Tr}(\rho(X) \rho(Y))$. The representation matrices satisfy the reality condition $\rho(X)^{*}=C^{-1} \rho(X) C$ with the 'charge conjugation' matrix

$$
C=V^{-1} W^{+}=W^{-1} V^{T}=\left(\begin{array}{ccc}
-\tilde{y} & 0 & 0 \\
0 & \hat{C} & 0 \\
0 & 0 & -\tilde{y}
\end{array}\right),
$$

The $2 n \times 2$ matrix $\mathbf{A}$ (and similarly $\boldsymbol{\alpha}, \boldsymbol{\beta}, \ldots$ ) satisfies the reality condition $\mathbf{A}=\hat{C} \mathbf{A}^{*} \tilde{y}$ and can be expressed in terms of the $4 n$ components of $A$, the precise form depending on the choice of $\hat{V}$ and $\hat{W}$. Choosing $\hat{V}=i \sigma_{1} \otimes \mathbf{1}_{n}$ and $\hat{W}=\sigma_{3} \otimes \mathbf{1}_{n}$ with $\hat{C}=-\sigma_{2} \otimes \mathbf{1}_{n}$, as well as $\mathbf{A}=\left(A_{(c)}, \hat{C} A_{(c)}^{*}\right)$ with $A_{(c)}=A_{(e)}+i A_{(m)}$, we obtain

$$
(\alpha \cdot y \beta) \mathbf{1}_{2}=\boldsymbol{\beta}^{+} \hat{V} \boldsymbol{\alpha}-\boldsymbol{\alpha}^{+} \hat{V} \boldsymbol{\beta}, \quad y=\left(\begin{array}{cc}
0 & 2 \sigma_{1} \otimes \mathbf{1}_{n} \\
-2 \sigma_{1} \otimes \mathbf{1}_{n} & 0
\end{array}\right),
$$

i.e. $c=2$ and $\eta=\sigma_{1} \otimes \mathbf{1}_{n}$, as well as the $4 n$-dimensional real representation $\bar{\rho}=\hat{\rho} \otimes \tilde{\rho}$ of $\bar{g}$ in the form

$\bar{\rho}: \lambda^{i} s_{i} \mapsto \hat{\lambda}^{i}\left(\begin{array}{cc}\operatorname{Re} \hat{R}_{i} & -\operatorname{Im} \hat{R}_{i} \\ \operatorname{Im} \hat{R}_{i} & \operatorname{Re} \hat{R}_{i}\end{array}\right)+\left(\begin{array}{cc}\frac{\tilde{\lambda}^{1}}{2} i \sigma_{2} & \frac{\tilde{\lambda}^{2}}{2} i \sigma_{2}+\frac{\tilde{\lambda}^{3}}{2} \mathbf{1}_{2} \\ \frac{\tilde{\lambda}^{2}}{2} i \sigma_{2}-\frac{\tilde{\lambda}^{3}}{2} \mathbf{1}_{2} & -\frac{\tilde{\lambda}^{1}}{2} i \sigma_{2}\end{array}\right) \otimes \mathbf{1}_{n}$.

Finally we obtain

$$
P=\rho(\pi)=\left(\begin{array}{ccc}
\Delta^{1 / 2} \mathbf{1}_{2} & 0 & 0 \\
\hat{P} \mathbf{A} & \hat{P} & 0 \\
\Delta^{-1 / 2}\left(\psi \mathbf{1}_{2}+\frac{1}{2} \mathbf{A}^{+} \hat{V} \mathbf{A}\right) & \Delta^{-1 / 2} \mathbf{A}^{+} \hat{V} & \Delta^{-1 / 2} \mathbf{1}_{2}
\end{array}\right),
$$

and $\rho(J)=\frac{1}{2} M^{-1} d M$ with $M=P^{+} D P$, and therefore

$$
<J, J>=\operatorname{Tr}(\rho(J) \rho(J))=\operatorname{Tr}(\hat{\rho}(\hat{J}) \hat{\rho}(\hat{J}))-\frac{2}{\Delta} \partial A_{(c)}^{+} \hat{M} \partial A_{(c)}+\frac{(\partial \Delta)^{2}+\omega^{2}}{\Delta^{2}},
$$

with $\hat{\rho}(\hat{J})=\frac{1}{2} \hat{M}^{-1} d \hat{M}, \hat{M}=\hat{P}^{+} \hat{P}$, and $\hat{M} \sigma_{1} \otimes \mathbf{1}_{n} \hat{M}=\sigma_{1} \otimes \mathbf{1}_{n}$. We can therefore proceed as in Section A.2, and express the 4-dimensional theory in terms of the symmetric matrices

$$
\tilde{\mu}=\sigma_{1} \otimes \mathbf{1}_{n}(\operatorname{Re} \hat{M})^{-1} \sigma_{1} \otimes \mathbf{1}_{n}, \quad \tilde{\nu}=\sigma_{1} \otimes \mathbf{1}_{n}(\operatorname{Re} \hat{M})^{-1} \operatorname{Im} \hat{M} .
$$




\section{A.5 $S p(2 n+2 ; \mathrm{R}) / U(n, 1)$}

These correspond to 4-dimensional theories with $k=n$ vector fields and the $n(n+1)$ scalars from $S p(2 n ; \mathbf{R}) / U(n)$. For $n=1$ this is again the Einstein-Maxwell-dilaton-axion theory [8].

Consider the action for the 4-dimensional theory with

$$
<\bar{J}, \bar{J}>_{\bar{g}}=\operatorname{Tr}\left(\hat{M}^{-1} \partial \hat{M} \hat{M}^{-1} \partial \hat{M}\right)+\operatorname{Tr}\left(\hat{M}^{-1} \partial \hat{N} \hat{M}^{-1} \partial \hat{N}\right),
$$

$c=1, \tilde{\mu}=\hat{M}, \tilde{\nu}=\hat{N}$, where the symmetric matrices $\hat{M}=\hat{P}^{T} \hat{P}$ and $\hat{N}$ describe scalar 'dilaton' fields and pseudo scalar 'axion' fields respectively. Choosing $\eta=\mathbf{S}_{n}$, where $\mathbf{S}_{n}$ is the $n$-dimensional 'skew diagonal unit matrix' with elements $\left(\mathbf{S}_{n}\right)_{i j}=\delta_{i+j, n+1}$, we obtain the 3 -dimensional theory with $\bar{M}=\bar{P}^{T} \bar{P}$, and

$$
\bar{P}=\left(\begin{array}{cc}
\hat{P} & 0 \\
\mathbf{S}_{n} \hat{P}^{T-1} \hat{N} & \mathbf{S}_{n} \hat{P}^{T-1} \mathbf{S}_{n}
\end{array}\right), \quad y=\left(\begin{array}{cc}
0 & \mathbf{S}_{n} \\
-\mathbf{S}_{n} & 0
\end{array}\right)
$$

The matrix $\bar{P}$ is the $S p(2 n ; \mathbf{R}) / U(n)$ coset representative $\bar{\pi}$ in the $2 n$ dimensional matrix representation

$$
\bar{\rho}: \bar{\pi} \mapsto \bar{\rho}(\bar{\pi})=\bar{P}, \quad y \bar{P}=\bar{P}^{T-1} y, \quad \tau(\bar{P})=\bar{P}^{T-1},
$$

with the 'symplectic metric' $y=-y^{T}=-y^{-1}$, and we can express the invariant scalar product on the Lie algebra $\operatorname{sl}(2 n ; \mathbf{R})$ in the form $\langle X, Y\rangle_{\bar{g}}=$ $2 \operatorname{Tr}(\bar{\rho}(X) \bar{\rho}(Y))$.

In order to exhibit the $S p(2 n+2 ; \mathbf{R}) / U(n, 1) \sigma$-model consider the $(2 n+$ 2)-dimensional matrix representation $\rho(X)$ of the element $X=\epsilon e+h \cdot \alpha+$ $\delta d+\lambda^{i} s_{i}+a \cdot \beta+\kappa k$ of the Lie algebra $\operatorname{sp}(2 n+2 ; \mathbf{R})$

$$
\rho(X)=\left(\begin{array}{ccc}
\frac{1}{2} \delta & \frac{1}{\sqrt{2}} \alpha^{T} y & \epsilon \\
\frac{1}{\sqrt{2}} \beta & \lambda^{i} \bar{\rho}\left(s_{i}\right) & \frac{1}{\sqrt{2}} \alpha \\
\kappa & -\frac{1}{\sqrt{2}} \beta^{T} y & -\frac{1}{2} \delta
\end{array}\right),
$$

with the symplectic metric $V$ such that $\rho(X)=-V^{-1} \rho^{T}(X) V$ and with the involutive automorphism $\rho(\tau(X))=-D^{-1} \rho^{T}(X) D$, where

$$
V=\left(\begin{array}{cc}
0 & \mathbf{S}_{n+1} \\
-\mathbf{S}_{n+1} & 0
\end{array}\right), \quad D=\left(\begin{array}{ccc}
1 & 0 & 0 \\
0 & -\mathbf{1}_{2 n} & 0 \\
0 & 0 & 1
\end{array}\right)
$$


and with the scalar product $\langle X, Y\rangle=2 \operatorname{Tr}(\rho(X) \rho(Y))$. Finally we obtain

$$
P=\rho(\pi)=\left(\begin{array}{ccc}
\sqrt{\Delta} & 0 & 0 \\
\frac{1}{\sqrt{2}} \bar{P} A & \bar{P} & 0 \\
\frac{1}{\sqrt{\Delta}} \psi & -\frac{1}{\sqrt{2 \Delta}} A^{T} y & \frac{1}{\sqrt{\Delta}}
\end{array}\right),
$$

and $\rho(J)=\frac{1}{2} M^{-1} d M$ with $M=P^{T} D P$.

\section{A.6 The General Procedure}

For the remaining cases of Table 1 the group $G$ is one of the exceptional groups $G_{2}, F_{4}, E_{6}, E_{7}$, or $E_{8}$. Some of these cases describe the bosonic sector of supergravity theories, e.g., $N=8$ supergravity in 4 dimensions with $\bar{G} / \bar{H}=E_{7(+7)} / S U(8)$ and $G / H=E_{8(+8)} / S O^{*}(16)$ [9]. Instead of discussing each of these cases in detail, we outline the general procedure and apply it to Case 6 with $G / H=G_{2(+2)} /(S U(1,1) \times S U(1,1)), \bar{G} / \bar{H}=S U(1,1) / U(1)$, and $k=2$ vector fields.

Given the $G / H$ and $\bar{G} / \bar{H} \sigma$-models in 3 and 4 dimensions, we know that the 4-dimensional theory has $k=\frac{1}{2}(\operatorname{dim}(G / H)-\operatorname{dim}(\bar{G} / \bar{H})-2)=$ $\frac{1}{4}(\operatorname{dim} G-\operatorname{dim} \bar{G}-3)$ vector fields. Parametrizing the Lie algebra of $G$ as described in Chapter 3, and choosing some $c>0$ we find that the electromagnetic potentials transform with a $2 k$-dimensional real representation $\bar{\rho}$ of $\bar{G}$ with

$$
\bar{\rho}: s_{i} \mapsto \bar{\rho}\left(s_{i}\right)=R_{i}=-y^{-1} R_{i}^{T} y, \quad y=c\left(\begin{array}{cc}
0 & \eta^{T} \\
-\eta & 0
\end{array}\right),
$$

where $\eta$ is an arbitrary orthogonal matrix and $\tau\left(R_{i}\right)=-R_{i}^{T}$. The representation matrices have therefore the structure

$$
R_{i}=\left(\begin{array}{cc}
A_{i} & B_{i} \eta^{T} \\
\eta C_{i} & -\eta A_{i}^{T} \eta^{T}
\end{array}\right)
$$

with symmetric $k \times k$ matrices $B$ and $C$. In our example with $\bar{G} / \bar{H}=$ $S U(1,1) / U(1) \sim S L(2) / S O(2)$ we choose $c=1$ and generators $\bar{d}, \bar{e}$, and $\bar{k}$ of $\bar{G}$ with the commutation relations

$$
[\bar{d}, \bar{e}]=\bar{e}, \quad[\bar{d}, \bar{k}]=-\bar{k}, \quad[\bar{e}, \bar{k}]=2 \bar{d},
$$

the automorphism

$$
\tau(\bar{e})=-\bar{k}, \quad \tau(\bar{d})=-\bar{d}, \quad \tau(\bar{k})=-\bar{e},
$$


and the 4-dimensional matrix representation of the Lie algebra element $X=$ $\bar{\epsilon} \bar{e}+\bar{\delta} \bar{d}+\bar{\kappa} \bar{k}$

$$
\bar{\rho}(X)=\left(\begin{array}{cccc}
\frac{3}{2} \bar{\delta} & \sqrt{3} \bar{\epsilon} & 0 & 0 \\
\sqrt{3} \bar{\kappa} & \frac{1}{2} \bar{\delta} & 2 \bar{\epsilon} & 0 \\
0 & 2 \bar{\kappa} & -\frac{1}{2} \bar{\delta} & \sqrt{3} \bar{\epsilon} \\
0 & 0 & \sqrt{3} \bar{\kappa} & -\frac{3}{2} \bar{\delta}
\end{array}\right), \quad \eta=\left(\begin{array}{cc}
0 & 1 \\
-1 & 0
\end{array}\right) .
$$

Next we compute the invariant scalar product $\left\langle s_{i}, s_{j}\right\rangle$, in our example $<X, X>=3 \bar{\delta}^{2}+12 \bar{\epsilon} \bar{\kappa}$, and use the identity $x^{i}<s_{i}, s_{j}>=-2 y R_{j}$ to determine the matrices $x^{i}$.

We would like to choose coset representatives $\bar{\pi}$ from a triangular group $\bar{T}$, and choose a basis for the representation $\bar{\rho}$, such that the generators of $\bar{T}$ are represented by matrices $R_{i}$ with $B_{i}=0$ (and $A_{i}$ suitably restricted). If that is possible, we obtain matrices $\bar{P}=\bar{\rho}(\bar{\pi})$ and $\bar{M}=\bar{\rho}(\bar{\mu})$

$$
\bar{P}=\left(\begin{array}{cc}
\tilde{P} & 0 \\
\eta \tilde{P}^{T-1} \tilde{\nu} & \eta \tilde{P}^{T-1} \eta^{T}
\end{array}\right), \quad \bar{M}=\bar{P}^{T} \bar{P}=\left(\begin{array}{cc}
\tilde{\mu}+\tilde{\nu} \tilde{\mu}^{-1} \tilde{\nu} & \tilde{\nu} \tilde{\mu}^{-1} \eta^{T} \\
\eta \tilde{\mu}^{-1} \tilde{\nu} & \eta \tilde{\mu}^{-1} \eta^{T}
\end{array}\right)
$$

with symmetric matrices $\tilde{\mu}=\tilde{P}^{T} \tilde{P}$ and $\tilde{\nu}$, and can then reconstruct the 4dimensional theory in terms of these matrices. All we really need is, however, a way to express the matrices $\bar{M}$ in terms of $\tilde{\mu}, \tilde{\nu}$, and $\eta$. In our example we choose $\bar{\pi}=e^{\ln \varphi \bar{d}} e^{\chi \bar{k}}$ represented by

$$
\bar{P}=\left(\begin{array}{cccc}
\varphi^{3 / 2} & 0 & 0 & 0 \\
\sqrt{3} \varphi^{1 / 2} \chi & \varphi^{1 / 2} & 0 & 0 \\
\sqrt{3} \varphi^{-1 / 2} \chi^{2} & 2 \varphi^{-1 / 2} \chi & \varphi^{-1 / 2} & 0 \\
\varphi^{-3 / 2} \chi^{3} & \sqrt{3} \varphi^{-3 / 2} \chi^{2} & \sqrt{3} \varphi^{-3 / 2} \chi & \varphi^{-3 / 2}
\end{array}\right),
$$

and finally obtain

$$
\tilde{\mu}=\left(\begin{array}{cc}
\varphi^{3}+3 \varphi \chi^{2} & \sqrt{3} \varphi \chi \\
\sqrt{3} \varphi \chi & \varphi
\end{array}\right), \quad \tilde{\nu}=\left(\begin{array}{cc}
2 \chi^{3} & \sqrt{3} \chi^{2} \\
\sqrt{3} \chi^{2} & 2 \chi
\end{array}\right)
$$

and

$$
<\bar{J}, \bar{J}>=3 \frac{(\partial \varphi)^{2}+(\partial \chi)^{2}}{\varphi^{2}} .
$$

In the following we will show for the remaining Cases 7-15 from Table 1, that the matrices $\bar{M}$ can indeed be expressed in terms of $\tilde{\mu}, \tilde{\nu}$, and $\eta$ as required. In most cases this can be achieved by choosing $\bar{P}$ in the form given above. Assume that there exist bases in the Lie algebra $\bar{g}$ and 
in the representation space for $\bar{\rho}$ such that for each $R_{i}$ at least one of the submatrices $B_{i}$ and $C_{i}$ is zero. We can then choose the triangular subgroup $\bar{T}$ for the coset representatives, generated by Lie algebra elements $s_{i}$ with $B_{i}=0$ and with $A_{i}$ suitably restricted. This is always possible if there exists a 'coset generator' $X=-\tau(X) \in \bar{g}$ such that all eigenvalues of $\bar{\rho}(X)$ are nonzero. The symmetric matrix $\bar{\rho}(X)$ can be diagonalized by an orthogonal transformation, and we can choose the eigenvectors with positive and negative eigenvalues as first and last $k$ elements respectively of a new basis in the representation space for $\bar{\rho}$, automatically preserving the structure of $y$. Next we can diagonalize $\operatorname{Ad} X$, which is symmetric provided we choose a basis for $\bar{g}$ with $<s_{i}, \tau\left(s_{j}\right)>=-\delta_{i j}$, such that $\left[X, s_{i}\right]=\xi_{i} s_{i}$. In these new bases $B_{i}=0$ except when $\xi_{i}>0$ and $C_{i}=0$ except when $\xi_{i}<0$ as desired. In the following we demonstrate the existence of such an $X$ for each of the Cases 7-9 and 11-15 of Table 1. For Case 10, where no such $X$ can be found, we will directly demonstrate how to express the matrices $\bar{M}$ in terms of symmetric matrices $\tilde{\mu}$ and $\tilde{\nu}$.

\section{A.6.1 The 14-Dimensional Representation of $S p(6 ; \mathrm{R})$}

In Case 7 of Table 1 there are 7 vector fields and the electromagnetic potentials transform under one of the two inequivalent 14-dimensional representations of $S p(6 ; \mathbf{R})$. Decomposing this representation with respect to the subgroup $S L(2) \otimes S L(2) \otimes S L(2)$ (using the isomorphism $S p(2 ; \mathbf{R})=S L(2)$ ) yields $2 \otimes 2 \otimes 2 \oplus 2 \otimes 1 \otimes 1 \oplus 1 \otimes 2 \otimes 1 \oplus 1 \otimes 1 \otimes 2$. Each of the three $S L(2)$ subgroups has a coset generator $\bar{d}_{i}$ with eigenvalues $\pm \frac{1}{2}$ in the 2-dimensional real representation denoted by 2 . Therefore, we can choose $X=\bar{d}_{1}+\bar{d}_{2}+\bar{d}_{3}$ with eigenvalues $\pm \frac{1}{2}$ and $\pm \frac{3}{2}$.

\section{A.6.2 The 20-Dimensional Representation of $A_{5}$}

In Cases 8-10 of Table 1 there are 10 vector fields and the electromagnetic potentials transform under the 20-dimensional representation of one of the noncompact forms $s l(6), s u(3,3)$, or $s u(5,1)$ of the Lie algebra $A_{5}$. As representation space we may take the totally antisymmetric 3 -index tensors $\varphi_{i j k}$, real for $s l(6)$ and subject to the reality condition $\bar{\varphi}^{i j k} \equiv \gamma^{i l} \gamma^{j m} \gamma^{k n}\left(\varphi_{l m n}\right)^{*}=$ $\frac{1}{6} \epsilon^{i j k l m n} \varphi_{l m n}$ for $s u(3,3)$ and $s u(5,1)$, where $\gamma^{i j}$ is the $s u(p, q)$ metric (chosen as $\gamma=\left(\begin{array}{cc}\mathbf{1}_{p} & 0 \\ 0 & -\mathbf{1}_{q}\end{array}\right)$ for simplicity).

For $s l(6)$ and $s u(3,3)$ we can decompose the representation with respect to the subalgebra $\operatorname{sl}(2) \oplus \operatorname{sl}(2) \oplus \operatorname{sl}(2)$ as in Subsection A.6.1 (using this time 
the isomorphism $s u(1,1)=s l(2))$. Distributing the three indices of $\varphi_{i j k}$ among the three $s l(2)$ subalgebras yields again the representations $2 \otimes 2 \otimes 2$, $2 \otimes 1 \otimes 1,1 \otimes 2 \otimes 1$, and $2 \otimes 1 \otimes 1$. Therefore, we can again choose $X=\bar{d}_{1}+\bar{d}_{2}+\bar{d}_{3}$ with eigenvalues $\pm \frac{1}{2}$ and $\pm \frac{3}{2}$.

This procedure does, however, not work for Case 10 with $s u(5,1)$, and it may even be impossible to bring the matrices $\bar{P}$ into 'block triagonal' form. We therefore have to analyze the matrices $\bar{M}$ in some detail. Let $\hat{\rho}$ be the 6 -dimensional complex representation of $s u(5,1)$ with

$$
\hat{\rho}: s_{i} \mapsto \hat{\rho}\left(s_{i}\right)=\hat{R}_{i}, \quad \hat{V} \hat{R}_{i}+\hat{R}_{i}^{+} \hat{V}=0, \quad \tau\left(\hat{R}_{i}\right)=-\hat{R}_{i}^{+},
$$

with the $s u(5,1)$ metric $\hat{V}=\hat{V}^{+}$; we choose

$$
\hat{V}=\left(\begin{array}{cc}
\mathbf{1}_{5} & 0 \\
0 & -1
\end{array}\right), \quad \lambda^{i} \hat{R}_{i}=\left(\begin{array}{cc}
a & b \\
b^{+} & -\operatorname{Tr} a
\end{array}\right),
$$

where $a=\left(a_{i}{ }^{j}\right), i, j=1, \ldots, 5$ is (the matrix representative of) an element of $u(5)$, and $b=\left(b_{i}\right), b^{+}=\left(\bar{b}^{j}\right)$ represent the coset generators. Next consider the action of $s u(5,1)$ on the totally antisymmetric 3-index tensors $\varphi_{i j k}$, choosing $\varphi_{i j} \equiv \varphi_{i j 6}$ and $\bar{\varphi}^{i j}=\left(\varphi_{i j}\right)^{*}$ as basis for this 20-dimensional representation

$$
\tilde{\rho}: s_{i} \mapsto \tilde{R}_{i}, \quad \tilde{V} \tilde{R}_{i}+\tilde{R}_{i}^{+} \tilde{V}=0, \quad \tilde{W} \tilde{R}_{i}+\tilde{R}_{i}^{T} \tilde{W}=0, \quad \tau\left(\tilde{R}_{i}\right)=-\tilde{R}_{i}^{+},
$$

with matrices

$$
\lambda^{i} \tilde{R}_{i}=\left(\begin{array}{cc}
a_{i j}{ }^{k l} & \bar{b}_{i j k l} \\
b^{i j k l} & \bar{a}^{i j}{ }_{k l}
\end{array}\right), \quad \tilde{V}=\left(\begin{array}{cc}
-i \mathbf{1}_{10} & 0 \\
0 & i \mathbf{1}_{10}
\end{array}\right), \quad \tilde{W}=\left(\begin{array}{cc}
0 & i \mathbf{1}_{10} \\
-i \mathbf{1}_{10} & 0
\end{array}\right),
$$

and matrix elements

$$
a_{i j}{ }^{k l}=2 a_{[i}^{[k} \delta_{j]}^{l]}-\delta_{[i}^{[k} \delta_{j]}^{l]} a_{m}^{m}=-\bar{a}^{k l}{ }_{i j}, \quad b^{i j k l}=\frac{1}{2} \epsilon^{i j k l m} b_{m}=\left(\bar{b}_{i j k l}\right)^{*} .
$$

We finally obtain the real 20-dimensional representation $\bar{\rho}$ of $s u(5,1)$ with $\operatorname{Re} \varphi_{i j}$ and $\operatorname{Im} \varphi_{i j}$ as new basis

$$
\bar{\rho}: s_{i} \mapsto \bar{\rho}\left(s_{i}\right)=R_{i}=S^{-1} \tilde{R}_{i} S, \quad \bar{V} R_{i}+R_{i}^{T} \bar{V}=0, \quad \tau\left(R_{i}\right)=-R_{i}^{T},
$$

with

$$
S=\frac{1}{\sqrt{2}}\left(\begin{array}{cc}
1 & i \\
1 & -i
\end{array}\right), \quad \bar{V}=S^{+} \tilde{V} S=S^{T} \tilde{W} S=\left(\begin{array}{cc}
0 & \mathbf{1}_{10} \\
-\mathbf{1}_{10} & 0
\end{array}\right),
$$

and therefore $\bar{\rho}(\bar{\pi})=\bar{P}$ with $\bar{V} \bar{P}=\bar{P}^{T-1} \bar{V}$, and $\bar{\rho}(\bar{\mu})=\bar{P}^{T} \bar{P}=\bar{M}$ with $\bar{M} \bar{V} \bar{M}=\bar{V}$. The last equation implies that $\bar{M}$ has the required form with symmetric matrices $\tilde{\mu}, \tilde{\nu}$ and with $\eta=\mathbf{1}_{10}$. 


\section{A.6.3 The 32-Dimensional Representation of $D_{6}$}

In Cases 11-13 of Table 1 there are 16 vector fields and the electromagnetic potentials transform under a 32-dimensional real 'spinor' representation of one of the noncompact forms $s o(6,6), s o^{*}(12)$, or $s o(10,2)$ of the Lie algebra $D_{6}$. The Lie algebra $D_{n}$ has two inequivalent $2^{n-1}$-dimensional 'chiral' spinor representations $S_{ \pm}^{n}$. For $s o(p, q)$ with $p=q(\bmod 8)$ they are both real, for $s o(p, q)$ with $p=q+4(\bmod 8)$ they are both pseudo real, for $s o(p, q)$ with $p=q \pm 2(\bmod 8)$ and for $s o^{*}(4 p+2)$ they are complex conjugate, whereas $S_{+}^{n}$ is real and $S_{-}^{n}$ is pseudo real for $s o^{*}(4 p)$. Decomposing the representations $S_{ \pm}^{p+q}$ with respect to the subalgebra $D_{p} \oplus D_{q}$ yields $S_{ \pm}^{p+q}=S_{+}^{p} \otimes S_{ \pm}^{q} \oplus S_{-}^{p} \otimes S_{\mp}^{q}$.

For $s o(6,6)$ and $s o(10,2)$ we use the subalgebra $s o(2,2) \oplus D_{4}$ with $D_{4}=$ $s o(4,4)$ and $D_{4}=s o(8)$ respectively and obtain $S_{+}^{6}=S_{+}^{2} \otimes S_{+}^{4} \oplus S_{-}^{2} \otimes S_{-}^{4}$ with real spinors $S_{ \pm}^{2}$ and $S_{ \pm}^{4}$. Using the isomorphism $s o(2,2)=\operatorname{sl}(2) \oplus \operatorname{sl}(2)$, we find $S_{+}^{2}=2 \otimes 1$ and $S_{-}^{2}=1 \otimes 2$. Therefore, we can choose $X=\bar{d}_{+}+\bar{d}_{-}$ with eigenvalues $\pm \frac{1}{2}$, where $\bar{d}_{ \pm}$are the coset generators of the two $\operatorname{sl}(2)$ subalgebras.

For $s o^{*}(12)$ we use the subalgebra $s o^{*}(4) \oplus s o^{*}(4) \oplus s o^{*}(4)$ and obtain $S_{+}^{6}=\sum S_{ \pm}^{2} \otimes S_{ \pm}^{2} \otimes S_{ \pm}^{2}$, where the sum contains all combinations with an even number of $S_{-}^{2}$. Using the isomorphism $s o^{*}(4)=s l(2) \oplus s u(2)$ we find $S_{+}^{2}=2 \otimes 1$ and $S_{-}^{2}=1 \otimes 2^{\prime}$, where $2^{\prime}$ is the 2 -dimensional pseudo real representation of $s u(2)$. Therefore, we can choose $X=\bar{d}_{1}+\bar{d}_{2}+\bar{d}_{2}$ with eigenvalues $\pm \frac{1}{2}$ and $\pm \frac{3}{2}$, where $\bar{d}_{i}$ are the coset generators of the three $\operatorname{sl}(2)$ subalgebras.

\section{A.6.4 The 56-Dimensional Representation of $\boldsymbol{E}_{7}$}

In Cases 14 and 15 of Table 1 there are 28 vector fields and the electromagnetic potentials transform under the 56-dimensional representation of one of the noncompact forms $E_{7(+7)}$ or $E_{7(-25)}$ of the Lie algebra $E_{7}$. We first decompose the representation with respect to the subalgebra $\operatorname{sl}(2)+D_{6}$, with $D_{6}=s o(6,6)$ for $E_{7(+7)}$ or $D_{6}=s o(10,2)$ for $E_{7(-25)}$, and obtain $2 \otimes V^{6} \oplus 1 \otimes S_{+}^{6}$, where $V^{6}$ denotes the vector representation of $D_{6}$. Further decomposing the representations of $D_{6}$ with respect to the subalgebra $\operatorname{sl}(2) \oplus \operatorname{sl}(2) \oplus D_{4}$ as in Subsection A.6.3 finally yields $V^{6}=2 \otimes 2 \otimes 1 \oplus 1 \otimes 1 \otimes V^{4}$. Therefore, we can choose $X=\bar{d}_{1}+\bar{d}_{2}+\bar{d}_{2}$ with eigenvalues $\pm \frac{1}{2}$ and $\pm \frac{3}{2}$, where $\bar{d}_{i}$ are again the coset generators of the three $s l(2)$ subalgebras. 


\section{References}

[1] Breitenlohner, P., Maison, D., and Gibbons, G.: Commun. Math. Phys. 120 (1987) 295-334.

[2] Julia, B.: in Proc. John Hopkins Workshop on Particle Theory, Baltimore, 1981.

[3] Breitenlohner, P., and Maison, D.: Ann. Inst. Henri Poincaré 46 (1987) 215-246.

[4] Heusler, M.: Phys. Rev. D 56 (1997) 961-973.

[5] Neugebauer, G., and Kramer, D.: Ann. d. Physik 24 (1969) 62-71;

Geroch, R.: J. Math. Phys. 12 (1971) 918-924.

[6] Harrison, K.: J. Math. Phys. 9 (1968) 1744-1752.

[7] Carter, B., in Black Holes, Proc. 1972 Les Houches Summer School, Eds. De Witt, C. and De Witt, B.S., Gordon and Breach, New York, 1973.

[8] Gal'tsov, D.V., and Kechkin, O.V.: Phys. Rev. D 50 (1994) 7394-7399;

Gal'tsov, D.V.: Phys. Rev. Lett. 74 (1995) 2863-2866.

[9] Markus, N., and Schwarz, J.H.: Nucl. Phys. B 228 (1983) 145-162. 